\title{
The Cherenkov Telescope Array: layout, design and performance
}

\section{Orel Gueta ${ }^{a, *}$ on behalf of the CTA Consortium and the CTA Observatory}

(a complete list of authors can be found at the end of the proceedings)

${ }^{a}$ Deutsches Elektronen-Synchrotron (DESY),

Platanenallee 6, Zeuthen, Germany

E-mail: orel.gueta@desy.de

The Cherenkov Telescope Array (CTA) will be the next generation very-high-energy gamma-ray observatory. CTA is expected to provide substantial improvement in accuracy and sensitivity with respect to existing instruments thanks to a tenfold increase in the number of telescopes and their state-of-the-art design. Detailed Monte Carlo simulations are used to further optimise the number of telescopes and the array layout, and to estimate the observatory performance using updated models of the selected telescope designs. These studies are presented in this contribution for the two CTA stations located on the island of La Palma (Spain) and near Paranal (Chile) and for different operation and observation conditions.

$37^{\text {th }}$ International Cosmic Ray Conference (ICRC 2021)

July 12th - 23rd, 2021

Online - Berlin, Germany

${ }^{*}$ Presenter 


\section{Introduction}

The Cherenkov Telescope Array (CTA) [1] will be the next generation very-high-energy (VHE; $10 \mathrm{GeV} \lesssim E \lesssim 100 \mathrm{TeV}$ ) gamma-ray observatory. CTA will be composed of more than 50 imaging atmospheric Cherenkov telescopes (IACTs) and will be built at two sites located on the island of La Palma (Spain; 17.89W, 28.76N; $2158 \mathrm{~m}$ altitude) and near Paranal (Chile; 70.32W, 24.68S; $2147 \mathrm{~m}$ altitude). IACTs detect gamma rays by recording the Cherenkov light induced by extensive air shower particles produced in the interaction of the gamma ray with particles in the atmosphere [2]. CTA will be sensitive to gamma rays in a wide energy range, from $20 \mathrm{GeV}$ to beyond $300 \mathrm{TeV}$, thanks to the use of three types of telescopes of varying sizes; namely the Large-Sized Telescopes (LSTs), Medium-Sized Telescopes (MSTs) and Small-Sized Telescopes (SSTs). Its sensitivity is expected to be about a factor of five to ten better than current IACTs across the entire energy range, providing the capability to e.g., perform deep surveys of various sky regions. The short-term sensitivity of CTA will be a few orders of magnitude better than that of Fermi-LAT. This would enable the measurement of very-fast variability in active galactic nuclei flares and increase the likelihood of detecting short-timescale transient phenomena like gamma-ray bursts or black-hole mergers. CTA is expected to provide a substantial improvement in angular and energy resolution. The angular resolution of CTA is expected to reach around one arc minute at high energies. This, together with the large field of view $\left(4.5^{\circ}-8.5^{\circ}\right)$, will enable detailed imaging of extended gamma-ray sources. The improved energy resolution, reaching around $5 \%$ at $1 \mathrm{TeV}$, will make it easier to measure spectral cutoffs and detect spectral features such as those expected from dark matter.

The telescope simulation models used in previous CTA performance studies [3] were updated and new estimates were derived. These estimates, together with the telescope layout optimisation process, are presented in this contribution. The number of telescopes considered here is not the final one and is subject to change.

\section{Simulation and analysis}

To optimise the CTA telescope layout and derive its performance, detailed Monte Carlo simulations were generated. The process starts with simulations of extensive air showers and the Cherenkov light they induce using the CORSIKA program (version 7.7100) [4]. The Cherenkov photons from the air shower are propagated through the telescopes using the sim_telarray simulation package (version 2020-06-28) [5]. Detailed simulation models of the CTA telescopes, are included in sim_telarray. These models were updated for the purpose of this study. The update process included:

- obtaining an updated atmospheric model for La Palma and geomagnetic field values for both sites;

- performing detailed ray-tracing simulations of optical elements to e.g., update the reference model used in sim_telarray to model the shadowing on the camera as a function of off-axis angle due to various telescope components.

- collecting lab and on-site prototype measurements of various telescope components simulated in sim_telarray; 
- tuning simulation parameters to fit the measurements where necessary;

- deriving appropriate trigger threshold levels;

- estimating the expected night-sky background light level in each pixel.

A summary of the samples simulated for each site, including the number of events per particle type per site and the energy ranges, is given in Table 1 . Three zenith angles $\left(20^{\circ}, 40^{\circ}\right.$ and $\left.60^{\circ}\right)$ and both north and south pointings were simulated, with all telescopes pointing parallel to each other.

\begin{tabular}{c|c|c}
\hline Particle type & Energy range $[\mathrm{TeV}]$ & Number of events \\
\hline Point source gamma & $0.003-330$ & $1 \mathrm{e} 9$ \\
Diffuse gamma & $0.003-330$ & $5 \mathrm{e} 9$ \\
Electron/Positron & $0.003-330$ & $1 \mathrm{e} 9$ \\
Proton & $0.006-600$ & $20 \mathrm{e} 9$ \\
\hline
\end{tabular}

Table 1: Number of events simulated per particle type and the corresponding energy ranges. The same number of events was simulated for both the northern and southern sites.

The telescope layouts for a larger number of telescopes on both sites were extensively optimised in a previous study [6]. In order to further optimise the layout with fewer telescopes as considered here, a total of 180 telescope positions were simulated in the southern site and 84 positions in the northern site. Out of those simulated positions, many telescope configurations were considered. Figure 1 shows one configuration for the northern site, the Alpha configuration to be built in the construction phase, and three configurations for the southern site. The three telescope layouts in the southern site shown in Figure 1 are the leading candidates to be selected for construction. A final decision on the layout is dependent on the number of telescopes to be built. Also shown are the positions of four LSTs not included in the simulations. Those will potentially be added to the array in the future.

The reconstruction and analysis of the simulation output were performed using the Eventdisplay [7] analysis software (version prod5_d20200702). ${ }^{1}$ The main steps of the analysis consist of waveform integration, image cleaning, stereoscopic reconstruction and cut optimisation. A detailed description of the reconstruction can be found elsewhere (see e.g., Ref. [8]).

All of the performance evaluations shown in Figures 2-4 are for point-like gamma-ray sources observed at a zenith angle of $20 \mathrm{deg}$, located either at the centre of the field of view (on-axis) or $3^{\circ}-4^{\circ}$ off axis as indicated in the figures. Results were averaged between the two north and south pointings. Further zenith angles, off-axis angles and night-sky background levels were taken into account in the layout optimisation, but are not shown here for reasons of brevity.

\section{Layout optimisation and performance}

The main criteria typically used to evaluate the performance of IACTs are effective collection area, angular resolution, energy resolution, residual background rate and differential sensitivity. All

\footnotetext{
${ }^{1}$ Dedicated CTA reconstruction software is currently under development and is expected to provide improved performance compared to the one presented here.
} 

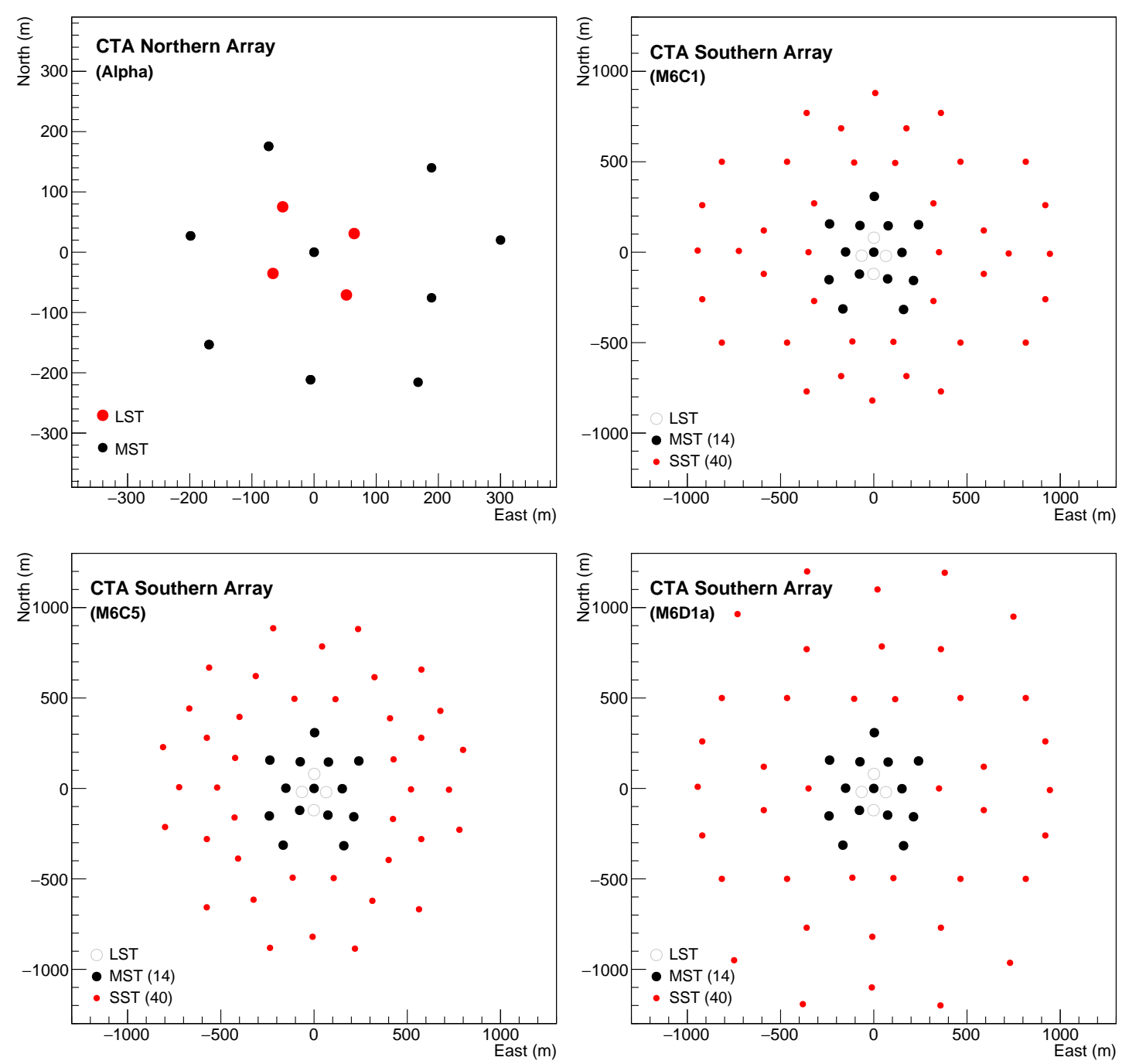

Figure 1: Telescope layouts for the CTA northern La Palma site (top left) and the southern Paranal site (top right, bottom). Telescope types are indicated in the legend. The Alpha layout shown for the northern site will be built during the construction phase. Three possible layouts are shown for the southern site, where the difference is in the layout of the SSTs. The LSTs shown in the southern layouts were not included in the simulations. They will potentially be added to the array in the future. Note that the area covered by the southern layout is approximately 19 times that of the northern layout.

were considered in the layout optimisation, but only the effective collection area, angular resolution and differential sensitivity are discussed here.

The differential sensitivity, defined as the minimal flux required to detect a point-like source $(\sigma>5)$, is the primary metric used to decide between telescope layouts. To calculate the sensitivity, apart from the detection requirement, at least ten detected gamma rays and a minimal signal to background ratio of 1/20 in each energy bin were required. The analysis cuts in each energy bin were optimised for best flux sensitivity in the northern site. In the southern site both flux sensitivity and angular resolution were taken into account in the optimisation process, with a higher weight 
given to the angular resolution. Figure 2 shows the on-axis sensitivity achieved with the various telescope layouts on both sites for an assumed 50 hour observation time (the optimal analysis cuts depend on the observation time). For the northern site, also the off-axis sensitivity is shown, where such off-axis observations at $3^{\circ}-4^{\circ}$ from the camera centre will be possible for the first time thanks to the large field of view of CTA cameras. The differential sensitivities of other instruments [9-16] are shown for comparison. These should provide only a rough comparison of the sensitivity of the different instruments, as the method of calculation and the criteria applied are not identical. The MAGIC and VERITAS sensitivities were combined by taking the more sensitive value of the two arrays in each bin and are represented by the IACT North curve.

A comparison between the effective collection areas of the three candidate layouts of the southern site are shown in the inset in Figure 2. The effective collection area is defined as the differential gamma-ray detection rate divided by the differential flux of incident gamma rays. The effective collection area was calculated assuming 30 minutes observation time and optimising the cuts for best sensitivity.
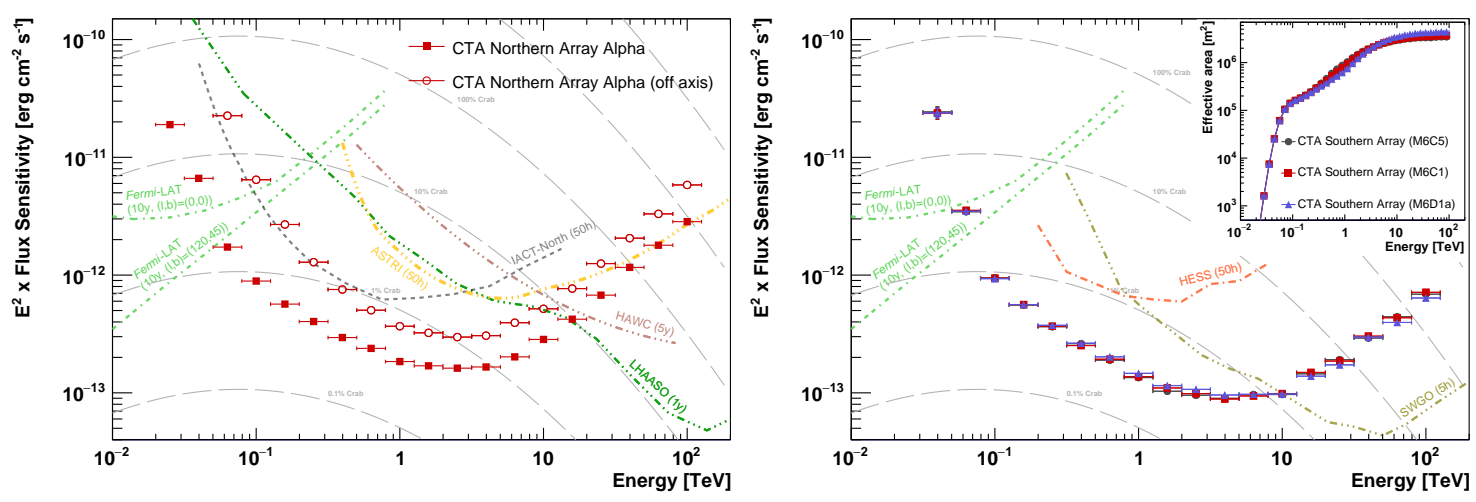

Figure 2: The CTA differential point-source sensitivity as a function of reconstructed energy in the northern array site (left) and for the various telescope layouts in the southern array site (right), assuming 50 hour observation time, pointing to 20 degrees zenith and averaged between pointing north and south. Filled markers represent observations assuming a gamma-ray source located at the centre of the field of view, while empty markers are for a source located $3^{\circ}-4^{\circ}$ off axis (northern site only). The differential sensitivities of other instruments [9-16] are shown for comparison. The IACT North curve represents the best-of sensitivity of MAGIC and VERITAS. The curves for Fermi-LAT and HAWC are scaled by a factor 1.2 relative those provided in the references, to account for the different energy binning. A comparison between the effective collection areas of the different telescope layouts in the south, calculated assuming 30 minutes observation time, is shown in the inset in the figure.

CTA will outperform current generation IACTs by about a factor five across the entire energy range. The energy coverage will expand as well, in particular at high energies, reaching hundreds of TeV, compared to $\sim 10 \mathrm{TeV}$ with current IACTs. Above $10-20 \mathrm{TeV}$, HAWC, LHAASO and SWGO are more sensitive than CTA, albeit with a worse angular resolution (at $100 \mathrm{TeV}$, the angular resolution of HAWC and SWGO is about $0.1^{\circ}$ and the LHAASO one is around $\left.0.2^{\circ}[14,16,17]\right)$.

In the southern site, a difference of about $10-20 \%$ in sensitivity is seen between the layouts. In particular, the M6D1a layout, where the SSTs are spread further apart (see the bottom right of Figure 1), is less sensitive around $5 \mathrm{TeV}$ and more sensitive above $10 \mathrm{TeV}$. This can be explained by the differences seen between the layouts in the effective collection area. 
The angular resolution as a function of the reconstructed energy obtained with the various telescope layouts on both sites is shown in Figure 3. The angular resolution in each energy bin is defined as the angle containing $68 \%$ of the reconstructed gamma-ray events relative to the simulated gamma-ray direction. A small improvement of less than $0.01^{\circ}$ in angular resolution is observed around $10 \mathrm{TeV}$ between the layouts in the southern site, where the angular resolution of the M6D1a layout is worse than of the other layouts. The angular resolution of other instruments $[9,11-$ 14, 16, 17] is shown in Figure 3 for comparison. CTA will provide a significant improvement in angular resolution compared to other instruments, ranging between $0.02^{\circ}$ to $0.2^{\circ}$. It should be noted that the analysis performed here was only partially optimised for best angular resolution. Better angular resolution is obtainable with appropriately optimised cuts in case of e.g., morphology studies of bright sources.
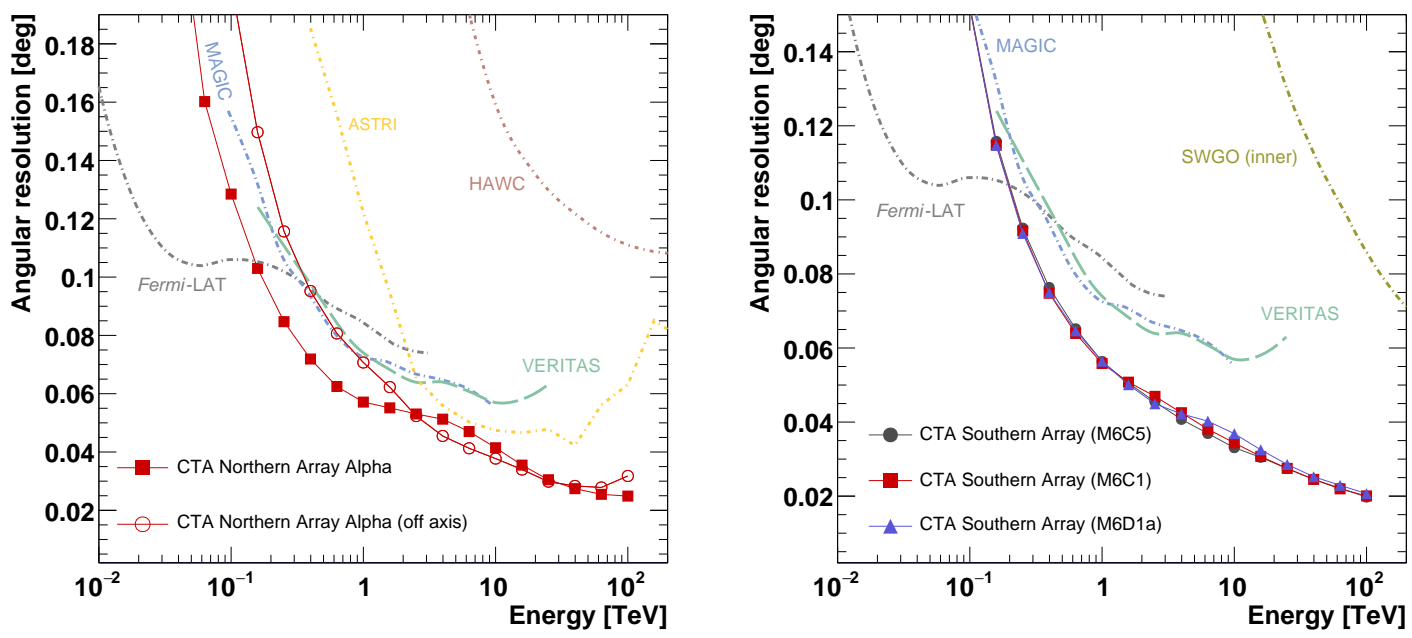

Figure 3: Angular resolution as a function of reconstructed energy for the northern (left) and southern site (right) of CTA. The angular resolution curves of the various layouts are indicated in the legend, where filled markers represent observations assuming a gamma-ray source located at the centre of the field of view, while empty markers are for a source located $3^{\circ}-4^{\circ}$ off axis (northern site only). The angular resolution of other instruments is shown for comparison $[9,11-14,16,17]$.

The short-transient phenomena discovery potential of the northern site of CTA is demonstrated in Figure 4, where the integral sensitivity in each energy bin for selected energies is shown as a function of observation time. The short-term sensitivity of Fermi-LAT is shown for comparison. CTA is a few orders of magnitude more sensitive than Fermi-LAT for such short observation times. The discovery potential of CTA for e.g., gamma-ray bursts, is therefore significantly higher than that of Fermi-LAT. However, that is true only for sources which are in the field of view. The Fermi-LAT field of view is substantially larger at $2.4 \mathrm{sr}$, making such occurrences much more likely. To enhance the efficiency of CTA in discovering short-term phenomena, CTA telescopes were designed with fast repointing capabilities. The LSTs will be able to repoint within 20 seconds of receiving an alert of a potential transient source. 


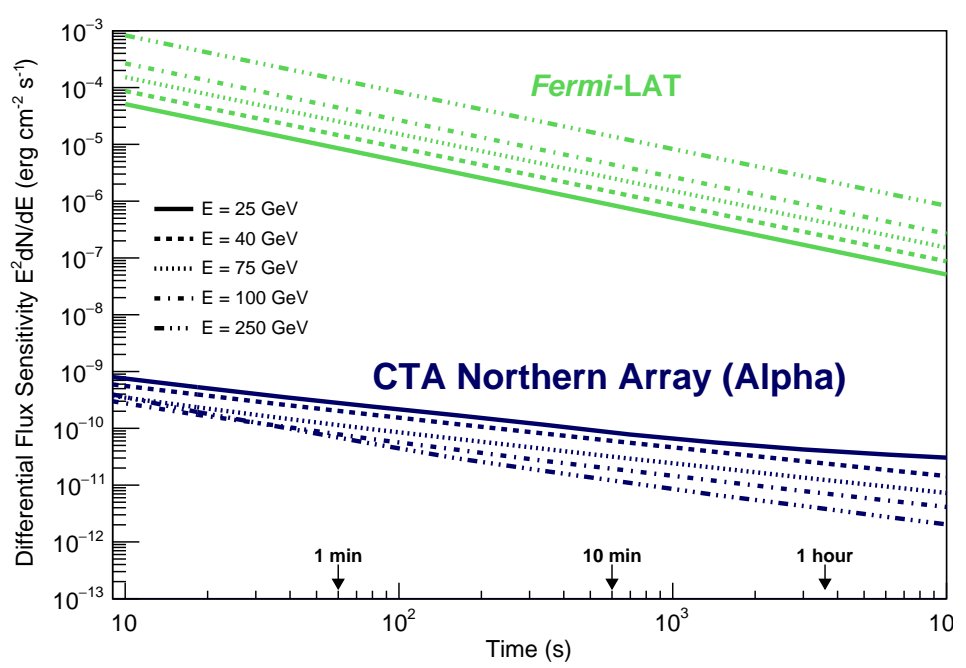

Figure 4: Differential flux sensitivity of the northern site of CTA at selected energies as function of observing time for 20 degrees zenith observation and averaged between pointing north and south, in comparison with Fermi-LAT (Pass 8 analysis, extragalactic background, standard survey observing mode).

\section{Conclusions}

The performance of various CTA telescope layouts was estimated and compared. The northern site of CTA is expected to be about five times more sensitive than MAGIC and VERITAS, while the southern site is expected to be an order of magnitude more sensitive than HESS. At energies above $10-30 \mathrm{TeV}$, CTA is less sensitive than water Cherenkov detector arrays, but provides significantly better angular and energy resolution. The differences between the sensitivities of the layouts in the southern site are of the order of $20 \%$ at energies above $1 \mathrm{TeV}$. The short-term sensitivity of CTA is expected to be a few orders of magnitude better than Fermi-LAT, making it a great instrument for short-term phenomena detections, in particular for serendipitous discoveries of sources in the field of view.

\section{Acknowledgments}

This work was conducted in the context of the CTA Consortium and CTA Observatory.

We gratefully acknowledge financial support from the agencies and organizations listed here: http://www. cta-observatory.org/consortium_acknowledgments. We also would like to thank the computing centres that provided resources for the generation of the instrument response functions, see Ref. [18] for a full list of contributing institutions.

\section{References}

[1] www.cta-observatory.org

[2] T. C. Weekes et al., 1989, ApJ, 342, 379. doi:10.1086/167599

[3] G. Maier et al., PoS ICRC2019, 733 doi:10.22323/1.358.0733 
[4] D. Heck et al., CORSIKA: a Monte Carlo code to simulate extensive air showers, 1998, Tech. Rep. FZKA 6019, Forschungszentrum Karlsruhe

[5] Bernlöhr, K., 2008, Astroparticle Physics, 30, 149

[6] Acharyya, A. et al. (The CTA Consortium), 2019, Astroparticle Physics 111, 35

[7] Maier, G. \& Holder, J., 2017, PoS (ICRC2017)747, arXiv:1708.04048

[8] Bernlohr, K. et al. Astropart. Physics 43, 171 (2013)

[9] https://www.slac.stanford.edu/exp/glast/groups/canda/lat_Performance. htm

[10] Holler et al. (The H.E.S.S. collaboration), 2015 Proceedings of the 34th ICRC (adapted)

[11] Aleksić, J. et al. (The MAGIC Collaboration), 2016 Astroparticle Physics 72, 76

[12] https://veritas.sao.arizona.edu/about-veritas/veritas-specifications (published 09/09/2009)

[13] S. Lombardi et al. (the ASTRI Project), 2021, PoS (ICRC2021) 884

[14] Abeysekara, A.U. et al. (The HAWC Collaboration), 2017, ApJ 843, 39 (World Scientific Publishing, 2019), ISBN 978-981-3270-08-4, arXiv: 1709.07997

[15] Cao Z., ISVHECRI, 2018, https: //indico.cern.ch/event/639198/contributions/ 2965294/attachments/1655868/2650647/ISVHECRI2018.pdf

[16] A. Albert, et al., arXiv:1902.08429

[17] F. Aharonian, et al., Chin. Phys. C 45 (2021) no.2, 025002 doi:10.1088/1674-1137/abd01b.

[18] https://www.cta-observatory.org/science/cta-performance 


\section{Full Authors List: the CTA Consortium and the CTA Observatory}

H. Abdalla ${ }^{1}$, H. Abe ${ }^{2}$, S. Abe ${ }^{2}$, A. Abusleme ${ }^{3}$, F. Acero ${ }^{4}$, A. Acharyya ${ }^{5}$, V. Acín Portella ${ }^{6}$, K. Ackley ${ }^{7}$, R. Adam ${ }^{8}$, C. Adams ${ }^{9}$, S.S. Adhikari ${ }^{10}$, I. Aguado-Ruesga ${ }^{11}$, I. Agudo ${ }^{12}$, R. Aguilera ${ }^{13}$, A. Aguirre-Santaella ${ }^{14}$, F. Aharonian ${ }^{15}$, A. Alberdi ${ }^{12}$, R. Alfaro ${ }^{16}$, J. Alfaro ${ }^{3}$, C. Alispach ${ }^{17}$, R. Aloisio ${ }^{18}$, R. Alves Batista ${ }^{19}$, J.-P. Amans ${ }^{20}$, L. Amati ${ }^{21}$, E. Amato ${ }^{22}$, L. Ambrogi ${ }^{18}$, G. Ambrosi ${ }^{23}$, M. Ambrosio ${ }^{24}$, R. Ammendola ${ }^{25}$, J. Anderson ${ }^{26}$, M. Anduze ${ }^{8}$, E.O. Angüner ${ }^{27}$, L.A. Antonelli ${ }^{28}$, V. Antonuccio ${ }^{29}$, P. Antoranz ${ }^{30}$,

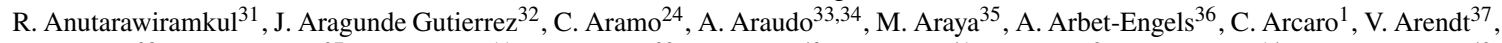
C. Armand ${ }^{38}$, T. Armstrong ${ }^{27}$, F. Arqueros ${ }^{11}$, L. Arrabito ${ }^{39}$, B. Arsioli ${ }^{40}$, M. Artero ${ }^{41}$, K. Asano ${ }^{2}$, Y. Ascasíbar ${ }^{14}$, J. Aschersleben $^{42}$, M. Ashley ${ }^{43}$, P. Attinà ${ }^{44}$, P. Aubert ${ }^{45}$, C. B. Singh ${ }^{19}$, D. Baack ${ }^{46}$, A. Babic ${ }^{47}$, M. Backes ${ }^{48}$, V. Baena ${ }^{13}$, S. Bajtlik ${ }^{49}$, A. Baktash ${ }^{50}$, C. Balazs ${ }^{7}$, M. Balbo ${ }^{38}$, O. Ballester ${ }^{41}$, J. Ballet ${ }^{4}$, B. Balmaverde ${ }^{44}$, A. Bamba ${ }^{51}$, R. Bandiera ${ }^{22}$, A. Baquero Larriva ${ }^{11}$, P. Barai ${ }^{19}$, C. Barbier ${ }^{45}$, V. Barbosa Martins ${ }^{52}$, M. Barcelo ${ }^{53}$, M. Barkov ${ }^{54}$, M. Barnard ${ }^{1}$, L. Baroncelli ${ }^{21}$, U. Barres de Almeida ${ }^{40}$, J.A. Barrio ${ }^{11}$, D. Bastieri ${ }^{55}$, P.I. Batista ${ }^{52}$, I. Batkovic ${ }^{55}$, C. Bauer ${ }^{53}$, R. Bautista-González ${ }^{56}$, J. Baxter ${ }^{2}$, U. Becciani ${ }^{29}$, J. Becerra González ${ }^{32}$, Y. Becherini ${ }^{57}$, G. Beck ${ }^{58}$, J. Becker Tjus ${ }^{59}$, W. Bednarek ${ }^{60}$, A. Belfiore ${ }^{61}$, L. Bellizzi ${ }^{62}$, R. Belmont ${ }^{4}$, W. Benbow ${ }^{63}$, D. Berge ${ }^{52}$, E. Bernardini ${ }^{52}$, M.I. Bernardos ${ }^{55}$, K. Bernlöhr ${ }^{53}$, A. Berti ${ }^{64}$, M. Berton ${ }^{65}$, B. Bertucci ${ }^{23}$, V. Beshley ${ }^{66}$, N. Bhatt ${ }^{67}$, S. Bhattacharyya ${ }^{67}$, W. Bhattacharyya ${ }^{52}$, S. Bhattacharyya ${ }^{68}$, B. Bi ${ }^{69}$, G. Bicknell $^{70}$, N. Biederbeck ${ }^{46}$, C. Bigongiari ${ }^{28}$, A. Biland ${ }^{36}$, R. Bird ${ }^{71}$, E. Bissaldi ${ }^{72}$,

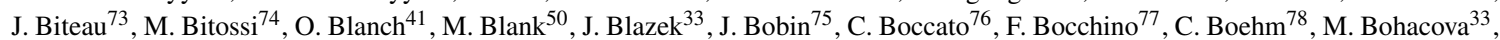
C. Boisson ${ }^{20}$, J. Boix ${ }^{41}$, J.-P. Bolle ${ }^{52}$, J. Bolmont ${ }^{79}$, G. Bonanno ${ }^{29}$, C. Bonavolontà ${ }^{24}$, L. Bonneau Arbeletche ${ }^{80}$, G. Bonnoli ${ }^{12}$, P. Bordas ${ }^{81}$, J. Borkowski ${ }^{49}$, S. Bórquez ${ }^{35}$, R. Bose ${ }^{82}$, D. Bose ${ }^{83}$, Z. Bosnjak ${ }^{47}$, E. Bottacini ${ }^{55}$, M. Böttcher ${ }^{1}$, M.T. Botticella ${ }^{84}$, C. Boutonnet ${ }^{85}$, F. Bouyjou ${ }^{75}$, V. Bozhilov ${ }^{86}$, E. Bozzo ${ }^{38}$, L. Brahimi ${ }^{39}$, C. Braiding ${ }^{43}$, S. Brau-Nogué ${ }^{87}$, S. Breen ${ }^{78}$, J. Bregeon ${ }^{39}$, M. Breuhaus ${ }^{53}$, A. Brill ${ }^{9}$, W. Brisken ${ }^{88}$, E. Brocato ${ }^{28}$, A.M. Brown ${ }^{5}$, K. Brügge ${ }^{46}$, P. Brun ${ }^{89}$, P. Brun ${ }^{39}$, F. Brun ${ }^{89}$, L. Brunetti ${ }^{45}$, G. Brunetti ${ }^{90}$, P. Bruno ${ }^{29}$, A. Bruno ${ }^{91}$, A. Bruzzese ${ }^{6}$, N. Bucciantini ${ }^{22}$, J. Buckley ${ }^{82}$, R. Bühler ${ }^{52}$, A. Bulgarelli ${ }^{21}$, T. Bulik ${ }^{92}$, M. Bünning ${ }^{52}$, M. Bunse ${ }^{46}$, M. Burton ${ }^{93}$, A. Burtovoi ${ }^{76}$, M. Buscemi ${ }^{94}$, S. Buschjäger ${ }^{46}$, G. Busetto ${ }^{55}$, J. Buss ${ }^{46}$, K. Byrum ${ }^{26}$, A. Caccianiga ${ }^{95}$, F. Cadoux ${ }^{17}$, A. Calanducci ${ }^{29}$, C. Calderón ${ }^{3}$, J. Calvo Tovar $^{32}$, R. Cameron ${ }^{96}$, P. Campaña ${ }^{35}$, R. Canestrari ${ }^{91}$, F. Cangemi ${ }^{79}$, B. Cantlay ${ }^{31}$, M. Capalbi ${ }^{91}$, M. Capasso ${ }^{9}$, M. Cappi ${ }^{21}$, A. Caproni ${ }^{97}$, R. Capuzzo-Dolcetta ${ }^{28}$, P. Caraveo ${ }^{61}$, V. Cárdenas ${ }^{98}$, L. Cardiel ${ }^{41}$, M. Cardillo ${ }^{99}$, C. Carlile ${ }^{100}$, S. Caroff ${ }^{45}$, R. Carosi ${ }^{74}$, A. Carosi ${ }^{17}$, E. Carquín ${ }^{35}$, M. Carrère ${ }^{39}$, J.-M. Casandjian ${ }^{4}$, S. Casanova ${ }^{101,53}$, E. Cascone ${ }^{84}$, F. Cassol ${ }^{27}$, A.J. Castro-Tirado ${ }^{12}$, F. Catalani ${ }^{102}$, O. Catalano ${ }^{91}$, D. Cauz ${ }^{103}$, A. Ceccanti ${ }^{64}$, C. Celestino Silva ${ }^{80}$, S. Celli ${ }^{18}$, K. Cerny ${ }^{104}$, M. Cerruti ${ }^{85}$, E. Chabanne ${ }^{45}$, P. Chadwick ${ }^{5}$, Y. Chai ${ }^{105}$, P. Chambery ${ }^{106}$, C. Champion ${ }^{85}$, S. Chandra ${ }^{1}$, S. Chaty ${ }^{4}$, A. Chen ${ }^{58}$, K. Cheng ${ }^{2}$, M. Chernyakova ${ }^{107}$, G. Chiaro ${ }^{61}$, A. Chiavassa ${ }^{64,108}$, M. Chikawa ${ }^{2}$, V.R. Chitnis ${ }^{109}$, J. Chudoba ${ }^{33}$, L. Chytka ${ }^{104}$, S. Cikota ${ }^{47}$, A. Circiello ${ }^{24110}$, P. Clark ${ }^{5}$, M. Çolak ${ }^{41}$, E. Colombo ${ }^{32}$, J. Colome ${ }^{13}$, S. Colonges ${ }^{85}$, A. Comastri ${ }^{21}$, A. Compagnino ${ }^{91}$, V. Conforti ${ }^{21}$, E. Congiu ${ }^{95}$, R. Coniglione ${ }^{94}$, J. Conrad $^{111}$, F. Conte ${ }^{53}$, J.L. Contreras ${ }^{11}$, P. Coppi ${ }^{112}$, R. Cornat ${ }^{8}$, J. Coronado-Blazquez ${ }^{14}$, J. Cortina ${ }^{113}$, A. Costa $^{29}$, H. Costantini ${ }^{27}$, G. Cotter ${ }^{114}$, B. Courty ${ }^{85}$, S. Covino ${ }^{95}$, S. Crestan ${ }^{61}$, P. Cristofari ${ }^{20}$, R. Crocker ${ }^{70}$, J. Croston ${ }^{115}$, K. Cubuk ${ }^{93}$, O. Cuevas ${ }^{98}$, X. Cui ${ }^{2}$, G. Cusumano ${ }^{91}$, S. Cutini2 ${ }^{23}$, A. D'Ai ${ }^{91}$, G. D’Amico ${ }^{116}$, F. D’Ammando ${ }^{90}$, P. D’Avanzo ${ }^{95}$, P. Da Vela ${ }^{74}$, M. Dadina ${ }^{21}$, S. Dai ${ }^{117}$, M. Dalchenko ${ }^{17}$, M. Dall' Ora ${ }^{84}$, M.K. Daniel ${ }^{63}$, J. Dauguet ${ }^{85}$, I. Davids ${ }^{48}$, J. Davies ${ }^{114}$, B. Dawson ${ }^{118}$, A. De Angelis ${ }^{55}$, A.E. de Araújo Carvalho ${ }^{40}$, M. de Bony de Lavergne ${ }^{45}$, V. De Caprio ${ }^{84}$, G. De Cesare ${ }^{21}$, F. De Frondat ${ }^{20}$, E.M. de Gouveia Dal Pino ${ }^{19}$, I. de la Calle ${ }^{11}$, B. De Lotto ${ }^{103}$, A. De Luca ${ }^{61}$, D. De Martino ${ }^{84}$, R.M. de Menezes $^{19}$, M. de Naurois ${ }^{8}$, E. de Oña Wilhelmi ${ }^{13}$, F. De Palma ${ }^{64}$, F. De Persio ${ }^{119}$, N. de Simone ${ }^{52}$, V. de Souza ${ }^{80}$, M. Del Santo ${ }^{91}$, M.V. del Valle ${ }^{19}$, E. Delagnes ${ }^{75}$, G. Deleglise ${ }^{45}$, M. Delfino Reznicek ${ }^{6}$, C. Delgado ${ }^{113}$, A.G. Delgado Giler ${ }^{80}$, J. Delgado Mengual ${ }^{6}$, R. Della Ceca ${ }^{95}$, M. Della Valle ${ }^{84}$, D. della Volpe ${ }^{17}$, D. Depaoli ${ }^{64,108}$, D. Depouez ${ }^{27}$, J. Devin ${ }^{85}$, T. Di Girolamo ${ }^{24,110}$, C. Di Giulio ${ }^{25}$, A. Di Piano ${ }^{21}$, F. Di Pierro ${ }^{64}$, L. Di Venere ${ }^{120}$, C. Díaz ${ }^{113}$, C. Díaz-Bahamondes ${ }^{3}$, C. Dib ${ }^{35}$, S. Diebold ${ }^{69}$, S. Digel ${ }^{96}$, R. Dima ${ }^{55}$, A. Djannati-Atai ${ }^{85}$, J. Djuvsland ${ }^{116}$, A. Dmytriiev ${ }^{20}$, K. Docher ${ }^{9}$, A. Domínguez ${ }^{11}$, D. Dominis Prester ${ }^{121}$, A. Donath ${ }^{53}$, A. Donini ${ }^{41}$, D. Dorner ${ }^{122}$, M. Doro ${ }^{55}$, R.d.C. dos Anjos ${ }^{123}$, J.-L. Dournaux ${ }^{20}$, T. Downes ${ }^{107}$, G. Drake ${ }^{26}$, H. Drass ${ }^{3}$, D. Dravins ${ }^{100}$, C. Duangchan ${ }^{31}$, A. Duara ${ }^{124}$, G. Dubus ${ }^{125}$, L. Ducci ${ }^{69}$, C. Duffy ${ }^{124}$, D. Dumora ${ }^{106}$, K. Dundas Mora ${ }^{111}$, A. Durkalec ${ }^{126}$, V.V. Dwarkadas ${ }^{127}$, J. Ebr $^{33}$, C. Eckner ${ }^{45}$, J. Eder ${ }^{105}$, A. Ederoclite ${ }^{19}$, E. Edy ${ }^{8}$, K. Egberts ${ }^{128}$, S. Einecke ${ }^{118}$, J. Eisch ${ }^{129}$, C. Eleftheriadis ${ }^{130}$, D. Elsässer ${ }^{46}$, G. Emery ${ }^{17}$, D. Emmanoulopoulos ${ }^{115}$, J.-P. Ernenwein ${ }^{27}$, M. Errando ${ }^{82}$, P. Escarate ${ }^{35}$, J. Escudero ${ }^{12}$, C. Espinoza ${ }^{3}$, S. Ettori ${ }^{21}$, A. Eungwanichayapant ${ }^{31}$, P. Evans ${ }^{124}$, C. Evoli ${ }^{18}$, M. Fairbairn ${ }^{131}$, D. Falceta-Goncalves ${ }^{132}$, A. Falcone ${ }^{133}$, V. Fallah Ramazani ${ }^{65}$, R. Falomo ${ }^{76}$, K. Farakos ${ }^{134}$, G. Fasola ${ }^{20}$, A. Fattorini ${ }^{46}$, Y. Favre ${ }^{17}$, R. Fedora ${ }^{135}$, E. Fedorova ${ }^{136}$, S. Fegan ${ }^{8}$, K. Feijen ${ }^{118}$, Q. Feng ${ }^{9}$, G. Ferrand ${ }^{54}$, G. Ferrara ${ }^{94}$, O. Ferreira ${ }^{8}$, M. Fesquet ${ }^{75}$, E. Fiandrini ${ }^{23}$, A. Fiasson ${ }^{45}$, M. Filipovic ${ }^{117}$, D. Fink ${ }^{105}$, J.P. Finley ${ }^{137}$, V. Fioretti ${ }^{21}$, D.F.G. Fiorillo ${ }^{24,110}$, M. Fiorini ${ }^{61}$, S. Flis ${ }^{52}$, H. Flores ${ }^{20}$, L. Foffano ${ }^{17}$, C. Föhr ${ }^{53}$, M.V. Fonseca ${ }^{11}$, L. Font ${ }^{138}$, G. Fontaine ${ }^{8}$, O. Fornieri ${ }^{52}$, P. Fortin ${ }^{63}$, L. Fortson ${ }^{88}$, N. Fouque ${ }^{45}$, A. Fournier ${ }^{106}$, B. Fraga ${ }^{40}$, A. Franceschini ${ }^{76}$, F.J. Franco ${ }^{30}$, A. Franco Ordovas ${ }^{32}$, L. Freixas Coromina ${ }^{113}$, L. Fresnillo ${ }^{30}$, C. Fruck ${ }^{105}$, D. Fugazza ${ }^{95}$, Y. Fujikawa ${ }^{139}$, Y. Fujita ${ }^{2}$, S. Fukami $^{2}$, Y. Fukazawa ${ }^{140}$, Y. Fukui ${ }^{141}$, D. Fulla ${ }^{52}$, S. Funk ${ }^{142}$, A. Furniss ${ }^{143}$, O. Gabella ${ }^{39}$, S. Gabici ${ }^{85}$, D. Gaggero ${ }^{14}$, G. Galanti ${ }^{61}$, G. Galaz $^{3}$, P. Galdemard ${ }^{144}$, Y. Gallant ${ }^{39}$, D. Galloway ${ }^{7}$, S. Gallozzi ${ }^{28}$, V. Gammaldi ${ }^{14}$, R. Garcia ${ }^{41}$, E. Garcia ${ }^{45}$, E. García ${ }^{13}$, R. Garcia López ${ }^{32}$, M. Garczarczyk ${ }^{52}$, F. Gargano ${ }^{120}$, C. Gargano ${ }^{91}$, S. Garozzo ${ }^{29}$, D. Gascon ${ }^{81}$, T. Gasparetto ${ }^{145}$, D. Gasparrini ${ }^{25}$, H. Gasparyan ${ }^{52}$, M. Gaug ${ }^{138}$, N. Geffroy ${ }^{45}$, A. Gent ${ }^{16}$, S. Germani ${ }^{76}$, L. Gesa ${ }^{13}$, A. Ghalumyan ${ }^{147}$, A. Ghedina ${ }^{148}$, G. Ghirlanda ${ }^{95}$, F. Gianotti ${ }^{21}$,

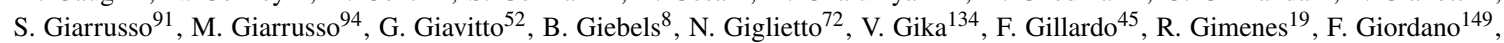
G. Giovannini ${ }^{90}$, E. Giro ${ }^{76}$, M. Giroletti ${ }^{90}$, A. Giuliani ${ }^{61}$, L. Giunti ${ }^{85}$, M. Gjaja ${ }^{9}$, J.-F. Glicenstein ${ }^{89}$, P. Gliwny ${ }^{60}$, N. Godinovic ${ }^{150}$, H. Göksu ${ }^{53}$, P. Goldoni ${ }^{85}$, J.L. Gómez ${ }^{12}$, G. Gómez-Vargas ${ }^{3}$, M.M. González ${ }^{16}$, J.M. González ${ }^{151}$, K.S. Gothe ${ }^{109}$, D. Götz ${ }^{4}$, J. Goulart Coelho $^{123}$, K. Gourgouliatos 5 , T. Grabarczyk ${ }^{152}$, R. Graciani ${ }^{81}$, P. Grandi ${ }^{21}$, G. Grasseau $^{8}$, D. Grasso ${ }^{74}$, A.J. Green ${ }^{78}$, D. Green ${ }^{105}$, J. Green ${ }^{28}$, T. Greenshaw ${ }^{153}$, I. Grenier ${ }^{4}$, P. Grespan ${ }^{55}$, A. Grillo ${ }^{29}$, M.-H. Grondin ${ }^{106}$, J. Grube ${ }^{131}$, V. Guarino ${ }^{26}$, B. Guest ${ }^{37}$, O. Gueta ${ }^{52}$, M. Gündüz ${ }^{59}$, S. Gunji ${ }^{154}$, A. Gusdorf ${ }^{20}$, G. Gyuk ${ }^{155}$, J. Hackfeld ${ }^{59}$, D. Hadasch ${ }^{2}$, J. Haga ${ }^{139}$, L. Hagge ${ }^{52}$, A. Hahn ${ }^{105}$,

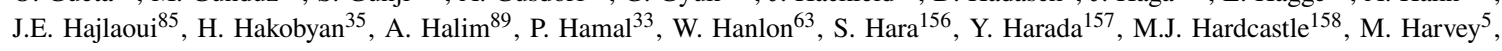


K. Hashiyama ${ }^{2}$, T. Hassan Collado ${ }^{113}$, T. Haubold ${ }^{105}$, A. Haupt ${ }^{52}$, U.A. Hautmann ${ }^{159}$, M. Havelka ${ }^{33}$, K. Hayashi ${ }^{141}$, K. Hayashi ${ }^{160}$, M. Hayashida ${ }^{161}$, H. He ${ }^{54}$, L. Heckmann ${ }^{105}$, M. Heller ${ }^{17}$, J.C. Helo ${ }^{35}$, F. Henault ${ }^{125}$, G. Henri ${ }^{125}$, G. Hermann ${ }^{53}$, R. Hermel ${ }^{45}$, S. Hernández Cadena ${ }^{16}$, J. Herrera Llorente ${ }^{32}$, A. Herrero ${ }^{32}$, O. Hervet ${ }^{143}$, J. Hinton ${ }^{53}$, A. Hiramatsu ${ }^{157}$, N. Hiroshima ${ }^{54}$, K. Hirotani $^{2}$, B. Hnatyk ${ }^{136}$, R. Hnatyk ${ }^{136}$, J.K. Hoang ${ }^{11}$, D. Hoffmann ${ }^{27}$, W. Hofmann ${ }^{53}$, C. Hoischen ${ }^{128}$, J. Holder ${ }^{162}$, M. Holler ${ }^{163}$, B. Hona ${ }^{164}$, D. Horan ${ }^{8}$, J. Hörandel ${ }^{165}$, D. Horns ${ }^{50}$, P. Horvath ${ }^{104}$, J. Houles ${ }^{27}$, T. Hovatta ${ }^{65}$, M. Hrabovsky ${ }^{104}$, D. Hrupec ${ }^{166}$, Y. Huang ${ }^{135}$, J.-M. Huet ${ }^{20}$, G. Hughes ${ }^{159}$, D. Hui ${ }^{2}$, G. Hull ${ }^{73}$, T.B. Humensky ${ }^{9}$, M. Hütten ${ }^{105}$, R. Iaria ${ }^{77}$, M. Iarlori ${ }^{18}$, J.M. Illa ${ }^{41}$, R. Imazawa ${ }^{140}$, D. Impiombato ${ }^{91}$, T. Inada ${ }^{2}$, F. Incardona ${ }^{29}$, A. Ingallinera ${ }^{29}$, Y. Inome ${ }^{2}$, S. Inoue ${ }^{54}$, T. Inoue ${ }^{141}$, Y. Inoue ${ }^{167}$, A. Insolia ${ }^{120,94}$, F. Iocco ${ }^{24,110}$, K. Ioka ${ }^{168}$, M. Ionica ${ }^{23}$, M. Iori ${ }^{119}$, S. Iovenitti ${ }^{95}$, A. Iriarte ${ }^{16}$, K. Ishio $^{105}$, W. Ishizaki ${ }^{168}$, Y. Iwamura ${ }^{2}$, C. Jablonski ${ }^{105}$, J. Jacquemier ${ }^{45}$, M. Jacquemont ${ }^{45}$, M. Jamrozy ${ }^{169}$, P. Janecek ${ }^{33}$, F. Jankowsky ${ }^{170}$, A. Jardin-Blicq ${ }^{31}$, C. Jarnot ${ }^{87}$, P. Jean ${ }^{87}$, I. Jiménez Martínez $^{113}$, W. Jin ${ }^{171}$, L. Jocou ${ }^{125}$, N. Jordana ${ }^{172}$, M. Josselin ${ }^{73}$, L. Jouvin ${ }^{41}$, I. Jung-Richardt ${ }^{142}$, F.J.P.A. Junqueira ${ }^{19}$, C. JuramyGilles $^{79}$, J. Jurysek ${ }^{38}$, P. Kaaret ${ }^{173}$, L.H.S. Kadowaki ${ }^{19}$, M. Kagaya ${ }^{2}$, O. Kalekin ${ }^{142}$, R. Kankanyan ${ }^{53}$, D. Kantzas ${ }^{174}$, V. Karas ${ }^{34}$, A. Karastergiou ${ }^{14}$, S. Karkar ${ }^{79}$, E. Kasai ${ }^{48}$, J. Kasperek ${ }^{175}$, H. Katagiri ${ }^{176}$, J. Kataoka ${ }^{177}$, K. Katarzyński ${ }^{178}$, S. Katsuda ${ }^{179}$, U. Katz ${ }^{142}$, N. Kawanaka ${ }^{180}$, D. Kazanas ${ }^{130}$, D. Kerszberg ${ }^{41}$, B. Khélifi ${ }^{85}$, M.C. Kherlakian ${ }^{52}$, T.P. Kian ${ }^{181}$, D.B. Kieda ${ }^{164}$, T. Kihm ${ }^{53}$, S. Kim ${ }^{3}$, S. Kimeswenger ${ }^{163}$, S. Kisaka ${ }^{140}$, R. Kissmann ${ }^{163}$, R. Kleijwegt ${ }^{135}$, T. Kleiner ${ }^{52}$, G. Kluge ${ }^{10}$, W. Kluźniak ${ }^{49}$, J. Knapp ${ }^{52}$, J. Knödlseder ${ }^{87}$, A. Kobakhidze ${ }^{78}$, Y. Kobayashi ${ }^{2}$, B. Koch ${ }^{3}$, J. Kocot ${ }^{152}$, K. Kohri ${ }^{182}$, K. Kokkotas ${ }^{69}$, N. Komin ${ }^{58}$, A. Kong ${ }^{2}$, K. Kosack ${ }^{4}$, G. Kowal ${ }^{132}$, F. Krack ${ }^{52}$, M. Krause ${ }^{52}$, F. Krennrich ${ }^{129}$, M. Krumholz ${ }^{70}$, H. Kubo ${ }^{180}$, V. Kudryavtsev ${ }^{183}$, S. Kunwar ${ }^{53}$, Y. Kuroda ${ }^{139}$, J. Kushida ${ }^{157}$, P. Kushwaha ${ }^{19}$, A. La Barbera ${ }^{91}$, N. La Palombara ${ }^{61}$, V. La Parola ${ }^{91}$, G. La Rosa ${ }^{91}$, R. Lahmann ${ }^{142}$, G. Lamanna ${ }^{45}$, A. Lamastra ${ }^{28}$, M. Landoni ${ }^{95}$, D. Landriu ${ }^{4}$, R.G. Lang ${ }^{80}$, J. Lapington ${ }^{124}$, P. Laporte ${ }^{20}$, P. Lason ${ }^{152}$, J. Lasuik ${ }^{37}$, J. Lazendic-Galloway ${ }^{7}$, T. Le Flour ${ }^{45}$, P. Le Sidane ${ }^{20}$, S. Leach ${ }^{124}$, A. Leckngam ${ }^{31}$, S.-H. Lee ${ }^{180}$, W.H. Lee ${ }^{16}$, S. Lee ${ }^{118}$, M.A. Leigui de Oliveira $^{184}$, A. Lemière ${ }^{85}$, M. Lemoine-Goumard ${ }^{106}$, J.-P. Lenain ${ }^{79}$, F. Leone ${ }^{94,185}$, V. Leray ${ }^{8}$, G. Leto ${ }^{29}$, F. Leuschner ${ }^{69}$, C. Levy ${ }^{79,20}$, R. Lindemann ${ }^{52}$, E. Lindfors ${ }^{65}$, L. Linhoff ${ }^{46}$, I. Liodakis ${ }^{65}$, A. Lipniacka ${ }^{116}$, S. Lloyd ${ }^{5}$, M. Lobo ${ }^{113}$, T. Lohse ${ }^{186}$, S. Lombardi ${ }^{28}$, F. Longo ${ }^{145}$, A. Lopez ${ }^{32}$, M. López ${ }^{11}$, R. López-Coto ${ }^{55}$, S. Loporchio ${ }^{149}$, F. Louis ${ }^{75}$, M. Louys ${ }^{20}$, F. Lucarelli ${ }^{28}$, D. Lucchesi ${ }^{55}$, H. Ludwig Boudi ${ }^{39}$, P.L. Luque-Escamilla ${ }^{56}$, E. Lyard ${ }^{38}$, M.C. Maccarone ${ }^{91}$, T. Maccarone ${ }^{187}$, E. Mach ${ }^{101}$, A.J. Maciejewski ${ }^{188}$, J. Mackey ${ }^{15}$, G.M. Madejski ${ }^{96}$, P. Maeght ${ }^{39}$, C. Maggio ${ }^{138}$, G. Maier ${ }^{52}$, A. Majczyna ${ }^{126}$, P. Majumdar ${ }^{83,2}$, M. Makariev ${ }^{189}$, M. Mallamaci ${ }^{55}$, R. Malta Nunes de Almeida ${ }^{184}$, S. Maltezos ${ }^{134}$, D. Malyshev ${ }^{142}$, D. Malyshev69, D. Mandat ${ }^{33}$, G. Maneva ${ }^{189}$, M. Manganaro ${ }^{121}$, G. Manicò ${ }^{94}$, P. Manigot ${ }^{8}$, K. Mannheim ${ }^{122}$, N. Maragos ${ }^{134}$, D. Marano ${ }^{29}$, M. Marconi ${ }^{84}$, A. Marcowith ${ }^{39}$, M. Marculewicz ${ }^{190}$, B. Marčun ${ }^{68}$, J. Marín $^{98}$, N. Marinello $^{55}$, P. Marinos ${ }^{118}$, M. Mariotti ${ }^{55}$, S. Markoff ${ }^{174}$, P. Marquez ${ }^{41}$, G. Marsella $^{94}$, J. Martí ${ }^{56}$, J.-M. Martin ${ }^{20}$, P. Martin ${ }^{87}$, O. Martinez ${ }^{30}$, M. Martínez ${ }^{41}$, G. Martínez ${ }^{113}$, O. Martínez ${ }^{41}$, H. Martínez-Huerta ${ }^{80}$, C. Marty $^{87}$, R. Marx ${ }^{53}$, N. Masetti ${ }^{21}, 151$, P. Massimino ${ }^{29}$, A. Mastichiadis ${ }^{191}$, H. Matsumoto ${ }^{167}$, N. Matthews ${ }^{164}$, G. Maurin ${ }^{45}$, W. Max-Moerbeck ${ }^{192}$, N. Maxted $^{43}$, D. Mazin²,105, M.N. Mazziotta ${ }^{120}$, S.M. Mazzola ${ }^{77}$, J.D. Mbarubucyeye ${ }^{52}$, L. Mc Comb ${ }^{5}$, I. McHardy ${ }^{115}$, S. McKeague ${ }^{107}$, S. McMuldroch ${ }^{63}$, E. Medina ${ }^{64}$, D. Medina Miranda ${ }^{17}$, A. Melandri ${ }^{95}$, C. Melioli1 ${ }^{19}$, D. Melkumyan ${ }^{52}$, S. Menchiari ${ }^{62}$, S. Mender ${ }^{46}$, S. Mereghetti ${ }^{61}$, G. Merino Arévalo ${ }^{6}$, E. Mestre ${ }^{13}$, J.-L. Meunier ${ }^{79}$, T. Meures ${ }^{135}$, M. Meyer ${ }^{142}$, S. Micanovic ${ }^{121}$, M. Miceli ${ }^{77}$, M. Michailidis ${ }^{69}$, J. Michałowski ${ }^{101}$, T. Miener ${ }^{11}$, I. Mievre ${ }^{45}$, J. Miller ${ }^{35}$, I.A. Minaya ${ }^{153}$, T. Mineo ${ }^{91}$, M. Minev ${ }^{189}$, J.M. Miranda ${ }^{30}$, R. Mirzoyan ${ }^{105}$, A. Mitchell ${ }^{36}$, T. Mizuno ${ }^{193}$, B. Mode ${ }^{135}$, R. Moderski $^{49}$, L. Mohrmann $^{142}$, E. Molina ${ }^{81}$, E. Molinari ${ }^{148}$, T. Montaruli ${ }^{17}$, I. Monteiro ${ }^{45}$, C. Moore ${ }^{124}$, A. Moralejo ${ }^{41}$, D. Morcuende-Parrilla ${ }^{11}$, E. Moretti ${ }^{41}$, L. Morganti ${ }^{64}$, K. Mori ${ }^{194}$, P. Moriarty ${ }^{15}$, K. Morik ${ }^{46}$, G. Morlino ${ }^{22}$, P. Morris ${ }^{14}$, A. Morselli ${ }^{25}$, K. Mosshammer ${ }^{52}$, P. Moya ${ }^{192}$, R. Mukherjee ${ }^{9}$, J. Muller $^{8}$, C. Mundell172, J. Mundet ${ }^{41}$, T. Murach ${ }^{52}$, A. Muraczewski ${ }^{49}$, H. Muraishi $^{195}$, K. Murase $^{2}$, I. Musella ${ }^{84}$, A. Musumarra ${ }^{120}$, A. Nagai ${ }^{17}$, N. Nagar ${ }^{196}$, S. Nagataki ${ }^{54}$, T. Naito ${ }^{156}$, T. Nakamori ${ }^{154}$, K. Nakashima ${ }^{142}$, K. Nakayama ${ }^{51}$, N. Nakhjiri ${ }^{13}$, G. Naletto ${ }^{55}$, D. Naumann ${ }^{52}$, L. Nava ${ }^{95}$, R. Navarro ${ }^{174}$, M.A. Nawaz ${ }^{132}$, H. Ndiyavala $^{1}$, D. Neise ${ }^{36}$, L. Nellen ${ }^{16}$, R. Nemmen ${ }^{19}$, M. Newbold ${ }^{164}$, N. Neyroud ${ }^{45}$, K. Ngernphat ${ }^{31}$, T. Nguyen Trung ${ }^{73}$, L. Nicastro ${ }^{21}$, L. Nickel ${ }^{46}$, J. Niemiec ${ }^{101}$, D. Nieto ${ }^{11}$, M. Nievas ${ }^{32}$, C. Nigro $^{41}$, M. Nikołajuk ${ }^{190}$, D. Ninci ${ }^{41}$, K. Nishijima ${ }^{157}$, K. Noda ${ }^{2}$, Y. Nogami $^{176}$, S. Nolan ${ }^{5}$, R. Nomura ${ }^{2}$, R. Norris ${ }^{117}$, D. Nosek ${ }^{197}$, M. Nöthe ${ }^{46}$, B. Novosyadlyj ${ }^{198}$, V. Novotny ${ }^{197}$, S. Nozaki ${ }^{180}$, F. Nunio ${ }^{144}$, P. O’Brien ${ }^{124}$, K. Obara ${ }^{176}$, R. Oger ${ }^{85}$, Y. Ohira ${ }^{51}$, M. Ohishi ${ }^{2}$, S. Ohm ${ }^{52}$, Y. Ohtani ${ }^{2}$, T. Oka ${ }^{180}$, N. Okazaki ${ }^{2}$, A. Okumura ${ }^{139,199}$, J.-F. Olive ${ }^{87}$, C. Oliver ${ }^{30}$, G. Olivera ${ }^{52}$, B. Olmi ${ }^{22}$, R.A. Ong ${ }^{71}$, M. Orienti ${ }^{90}$, R. Orito ${ }^{200}$, M. Orlandini ${ }^{21}$, S. Orlando ${ }^{77}$, E. Orlando ${ }^{145}$, J.P. Osborne ${ }^{124}$, M. Ostrowski ${ }^{169}$, N. Otte ${ }^{146}$, E. Ovcharov ${ }^{86}$, E. Owen ${ }^{2}$, I. Oya ${ }^{159}$, A. Ozieblo ${ }^{152}$, M. Padovani ${ }^{22}$, I. Pagano ${ }^{29}$, A. Pagliaro ${ }^{91}$, A. Paizis ${ }^{61}$, M. Palatiello ${ }^{145}$, M. Palatka ${ }^{33}$, E. Palazzi ${ }^{21}$, J.-L. Panazol ${ }^{45}$, D. Paneque ${ }^{105}$, B. Panes ${ }^{3}$, S. Panny ${ }^{163}$, F.R. Pantaleo ${ }^{72}$, M. Panter ${ }^{53}$, R. Paoletti ${ }^{62}$, M. Paolillo ${ }^{24,110}$, A. Papitto ${ }^{28}$, A. Paravac ${ }^{122}$, J.M. Paredes ${ }^{81}$, G. Pareschi ${ }^{95}$, N. Park ${ }^{127}$, N. Parmiggiani ${ }^{21}$, R.D. Parsons ${ }^{186}$, P. Paśko ${ }^{201}$, S. Patel ${ }^{52}$, B. Patricellii ${ }^{28}$, G. Pauletta ${ }^{103}$, L. Pavletić ${ }^{121}$, S. Pavy ${ }^{8}$, A. Pe'er ${ }^{105}$, M. Pech ${ }^{33}$, M. Pecimotika ${ }^{121}$, M.G. Pellegriti ${ }^{120}$, P. Peñil Del Campo $^{11}$, M. Penno ${ }^{52}$, A. Pepato ${ }^{55}$, S. Perard ${ }^{106}$, C. Perennes ${ }^{55}$, G. Peres ${ }^{77}$, M. Peresano ${ }^{4}$, A. Pérez-Aguilera ${ }^{11}$, J. Pérez-Romero ${ }^{14}$, M.A. Pérez-Torres ${ }^{12}$, M. Perri ${ }^{28}$, M. Persic ${ }^{103}$, S. Petrera ${ }^{18}$, P.-O. Petrucci ${ }^{125}$, O. Petruk ${ }^{66}$, B. Peyaud ${ }^{89}$, K. Pfrang ${ }^{52}$, E. Pian ${ }^{21}$, G. Piano $^{99}$, P. Piatteli ${ }^{94}$, E. Pietropaolo ${ }^{18}$, R. Pillera ${ }^{149}$, B. Pilszyk ${ }^{101}$, D. Pimentel ${ }^{202}$, F. Pintore ${ }^{91}$, C. Pio García ${ }^{41}$, G. Pirola ${ }^{64}$, F. Piron ${ }^{39}$, A. Pisarski ${ }^{190}$, S. Pita ${ }^{85}$, M. Pohl ${ }^{128}$, V. Poireau ${ }^{45}$, P. Poledrelli ${ }^{159}$, A. Pollo ${ }^{126}$, M. Polo ${ }^{113}$, C. Pongkitivanichkul ${ }^{31}$,

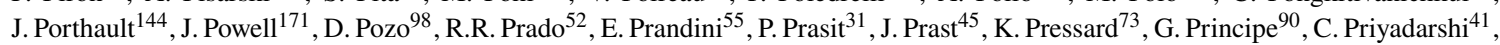
N. Produit ${ }^{38}$, D. Prokhorov ${ }^{174}$, H. Prokoph $^{52}$, M. Prouza ${ }^{33}$, H. Przybilski ${ }^{101}$, E. Pueschel ${ }^{52}$, G. Pühlhofer ${ }^{69}$, I. Puljak ${ }^{150}$, M.L. Pumo ${ }^{94}$, M. Punch ${ }^{85,57}$, F. Queiroz ${ }^{203}$, J. Quinn ${ }^{204}$, A. Quirrenbach ${ }^{170}$, S. Rainò ${ }^{149}$, P.J. Rajda ${ }^{175}$, R. Rando ${ }^{55}$, S. Razzaque ${ }^{205}$, E. Rebert ${ }^{20}$, S. Recchia ${ }^{85}$, P. Reichherzer ${ }^{59}$, O. Reimer ${ }^{163}$, A. Reimer ${ }^{163}$, A. Reisenegger ${ }^{3,206}$, Q. Remy ${ }^{53}$, M. Renaud ${ }^{39}$, T. Reposeur ${ }^{106}$, B. Reville ${ }^{53}$, J.-M. Reymond ${ }^{75}$, J. Reynolds ${ }^{15}$, W. Rhode ${ }^{46}$, D. Ribeiro ${ }^{9}$, M. Ribó ${ }^{81}$, G. Richards ${ }^{162}$, T. Richtler ${ }^{196}$, J. Rico ${ }^{41}$, F. Rieger ${ }^{53}$, L. Riitano ${ }^{135}$, V. Ripepi ${ }^{84}$, M. Riquelme ${ }^{192}$, D. Riquelme ${ }^{35}$, S. Rivoire ${ }^{39}$, V. Rizi ${ }^{18}$, E. Roache ${ }^{63}$, B. Röben ${ }^{159}$, M. Roche ${ }^{106}$, J. Rodriguez ${ }^{4}$, G. Rodriguez Fernandez ${ }^{25}$, J.C. Rodriguez Ramirez ${ }^{19}$, J.J. Rodríguez Vázquez ${ }^{113}$, F. Roepke ${ }^{170}$, G. Rojas $^{207}$, L. Romanato ${ }^{55}$, P. Romano ${ }^{95}$, G. Romeo ${ }^{29}$, F. Romero Lobato ${ }^{11}$, C. Romoli ${ }^{53}$, M. Roncadelli ${ }^{103}$, S. Ronda ${ }^{30}$, J. Rosado ${ }^{11}$, A. Rosales de Leon $^{5}$, G. Rowell ${ }^{118}$, B. Rudak ${ }^{49}$, A. Rugliancich ${ }^{74}$, J.E. Ruíz del Mazo ${ }^{12}$, W. Rujopakarn ${ }^{31}$, C. Rulten ${ }^{5}$, C. Russell ${ }^{3}$, 
F. Russo ${ }^{21}$, I. Sadeh ${ }^{52}$, E. Sæther Hatlen ${ }^{10}$, S. Safi-Harb ${ }^{37}$, L. Saha ${ }^{11}$, P. Saha ${ }^{208}$, V. Sahakian ${ }^{147}$, S. Sailer ${ }^{53}$, T. Saito ${ }^{2}$, N. Sakaki ${ }^{54}$, S. Sakurai ${ }^{2}$, F. Salesa Greus ${ }^{101}$, G. Salina ${ }^{25}$, H. Salzmann ${ }^{69}$, D. Sanchez ${ }^{45}$, M. Sánchez-Conde ${ }^{14}$, H. Sandaker ${ }^{10}$, A. Sandoval ${ }^{16}$, P. Sangiorgi ${ }^{91}$, M. Sanguillon ${ }^{39}$, H. Sano ${ }^{2}$, M. Santander ${ }^{171}$, A. Santangelo ${ }^{69}$, E.M. Santos ${ }^{202}$, R. Santos-Lima ${ }^{19}$, A. Sanuy ${ }^{81}$, L. Sapozhnikov ${ }^{96}$, T. Saric ${ }^{150}$, S. Sarkar ${ }^{114}$, H. Sasaki ${ }^{157}$, N. Sasaki ${ }^{179}$, K. Satalecka ${ }^{52}$, Y. Sato ${ }^{209}$, F.G. Saturni ${ }^{28}$, M. Sawada ${ }^{54}$, U. Sawangwit ${ }^{31}$, J. Schaefer ${ }^{142}$, A. Scherer ${ }^{3}$, J. Scherpenberg ${ }^{105}$, P. Schipani ${ }^{84}$, B. Schleicher ${ }^{122}$, J. Schmoll ${ }^{5}$, M. Schneider ${ }^{143}$, H. Schoorlemmer ${ }^{53}$, P. Schovanek ${ }^{33}$, F. Schussler ${ }^{89}$, B. Schwab ${ }^{142}$, U. Schwanke ${ }^{186}$, J. Schwarz ${ }^{95}$, T. Schweizer ${ }^{105}$, E. Sciacca ${ }^{29}$, S. Scuderi ${ }^{61}$, M. Seglar Arroyo ${ }^{45}$, A. Segreto ${ }^{91}$, I. Seitenzahl ${ }^{43}$, D. Semikoz ${ }^{85}$, O. Sergijenko ${ }^{136}$, J.E. Serna Franco ${ }^{16}$, M. Servillat ${ }^{20}$, K. Seweryn ${ }^{201}$, V. Sguera ${ }^{21}$, A. Shalchi ${ }^{37}$, R.Y. Shang ${ }^{71}$, P. Sharma ${ }^{73}$, R.C. Shellard ${ }^{40}$, L. Sidoli ${ }^{61}$, J. Sieiro ${ }^{81}$, H. Siejkowski ${ }^{152}$, J. Silk ${ }^{114}$, A. Sillanpää 65 , B.B. Singh ${ }^{109}$, K.K. Singh ${ }^{210}$, A. Sinha ${ }^{39}$, C. Siqueira ${ }^{80}$, G. Sironi ${ }^{55}$, J. Sitarek ${ }^{60}$, P. Sizun ${ }^{75}$, V. Sliusar ${ }^{38}$, A. Slowikowska ${ }^{178}$, D. Sobczyńska ${ }^{60}$, R.W. Sobrinho ${ }^{184}$, H. Sol $^{20}$, G. Sottile ${ }^{91}$, H. Spackman ${ }^{114}$, A. Specovius ${ }^{142}$, S. Spencer ${ }^{114}$, G. Spengler ${ }^{186}$, D. Spiga ${ }^{95}$, A. Spolon ${ }^{55}$, W. Springer ${ }^{164}$, A. Stamerra ${ }^{28}$, S. Stanič ${ }^{68}$, R. Starling ${ }^{124}$, Ł. Stawarz ${ }^{169}$, R. Steenkamp ${ }^{48}$, S. Stefanik ${ }^{197}$, C. Stegmann ${ }^{128}$, A. Steiner ${ }^{52}$, S. Steinmass ${ }^{53}$, C. Stella ${ }^{103}$, C. Steppa ${ }^{128}$, R. Sternberger ${ }^{52}$, M. Sterzel ${ }^{152}$, C. Stevens ${ }^{135}$, B. Stevenson ${ }^{71}$, T. Stolarczyk ${ }^{4}$, G. Stratta ${ }^{21}$, U. Straumann ${ }^{208}$, J. Strišković ${ }^{166}$, M. Strzys ${ }^{2}$, R. Stuik ${ }^{174}$, M. Suchenek ${ }^{211}$, Y. Suda ${ }^{140}$, Y. Sunada ${ }^{179}$, T. Suomijarvi ${ }^{73}$, T. Suric ${ }^{212}$, P. Sutcliffe ${ }^{153}$, H. Suzuki $^{213}$, P. Świerk ${ }^{101}$, T. Szepieniec ${ }^{152}$, A. Tacchini ${ }^{21}$, K. Tachihara ${ }^{141}$, G. Tagliaferri ${ }^{95}$, H. Tajima ${ }^{139}$, N. Tajima ${ }^{2}$, D. Tak ${ }^{52}$, K. Takahashi $^{214}$, H. Takahashi ${ }^{140}$, M. Takahashi ${ }^{2}$, M. Takahashi ${ }^{2}$, J. Takata $^{2}$,

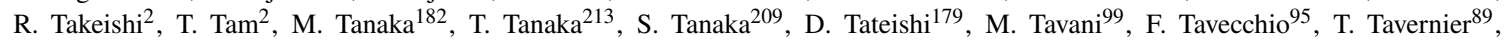

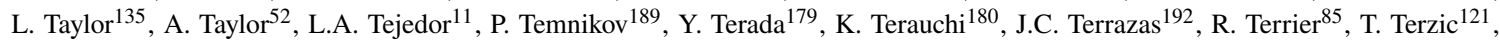

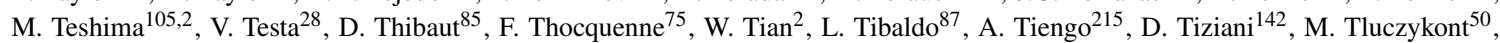
C.J. Todero Peixoto ${ }^{102}$, F. Tokanai ${ }^{154}$, K. Toma ${ }^{160}$, L. Tomankova ${ }^{142}$, J. Tomastik ${ }^{104}$, D. Tonev ${ }^{189}$, M. Tornikoski ${ }^{216}$, D.F. Torres ${ }^{13}$, E. Torresi ${ }^{21}$, G. Tosti ${ }^{95}$, L. Tosti ${ }^{23}$, T. Totani ${ }^{51}$, N. Tothill ${ }^{117}$, F. Toussenel ${ }^{79}$, G. Tovmassian ${ }^{16}$, P. Travnicek ${ }^{33}$, C. Trichard ${ }^{8}$,

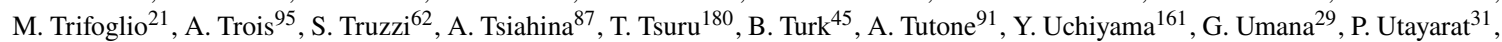
L. Vaclavek ${ }^{104}$, M. Vacula ${ }^{104}$, V. Vagelli ${ }^{23,217}$, F. Vagnetti ${ }^{25}$, F. Vakili ${ }^{218}$, J.A. Valdivia ${ }^{192}$, M. Valentino ${ }^{24}$, A. Valio ${ }^{19}$, B. Vallage ${ }^{89}$, P. Vallania ${ }^{44,64}$, J.V. Valverde Quispe ${ }^{8}$, A.M. Van den Berg ${ }^{42}$, W. van Driel ${ }^{20}$, C. van Eldik ${ }^{142}$, C. van Rensburg ${ }^{1}$, B. van Soelen ${ }^{210}$, J. Vandenbroucke ${ }^{135}$, J. Vanderwalt ${ }^{1}$, G. Vasileiadis ${ }^{39}$, V. Vassiliev ${ }^{71}$, M. Vázquez Acosta ${ }^{32}$, M. Vecchi ${ }^{42}$, A. Vega ${ }^{98}$, J. Veh $^{142}$, P. Veitch ${ }^{118}$, P. Venault ${ }^{75}$, C. Venter ${ }^{1}$, S. Ventura ${ }^{62}$, S. Vercellone ${ }^{95}$, S. Vergani ${ }^{20}$, V. Verguilov ${ }^{189}$, G. Verna ${ }^{27}$, S. Vernetto ${ }^{44,64}$, V. Verzi ${ }^{25}$, G.P. Vettolani ${ }^{90}$, C. Veyssiere ${ }^{144}$, I. Viale ${ }^{55}$, A. Viana ${ }^{80}$, N. Viaux ${ }^{35}$, J. Vicha ${ }^{33}$, J. Vignatti ${ }^{35}$, C.F. Vigorito ${ }^{64,108}$, J. Villanueva ${ }^{98}$, J. Vink ${ }^{174}$, V. Vitale ${ }^{23}$, V. Vittorini ${ }^{99}$, V. Vodeb ${ }^{68}$, H. Voelk $^{53}$, N. Vogel ${ }^{142}$, V. Voisin ${ }^{79}$, S. Vorobiov ${ }^{68}$, I. Vovk ${ }^{2}$, M. Vrastil ${ }^{33}$, T. Vuillaume ${ }^{45}$, S.J. Wagner ${ }^{170}$, R. Wagner ${ }^{105}$, P. Wagner $^{52}$, K. Wakazono ${ }^{139}$, S.P. Wakely ${ }^{127}$, R. Walter ${ }^{38}$, M. Ward ${ }^{5}$, D. Warren ${ }^{54}$, J. Watson ${ }^{52}$, N. Webb ${ }^{87}$, M. Wechakama ${ }^{31}$, P. Wegner ${ }^{52}$, A. Weinstein ${ }^{129}$, C. Weniger ${ }^{174}$, F. Werner ${ }^{53}$, H. Wetteskind $^{105}$, M. White ${ }^{118}$, R. White ${ }^{53}$, A. Wierzcholska ${ }^{101}$, S. Wiesand ${ }^{52}$, R. Wijers ${ }^{174}$, M. Wilkinson ${ }^{124}$, M. Will ${ }^{105}$, D.A. Williams ${ }^{143}$, J. Williams ${ }^{124}$, T. Williamson ${ }^{162}$, A. Wolter ${ }^{95}$, Y.W. Wong ${ }^{142}$, M. Wood ${ }^{96}$, C. Wunderlich ${ }^{62}$, T. Yamamoto ${ }^{213}$, H. Yamamoto ${ }^{141}$, Y. Yamane ${ }^{141}$, R. Yamazaki ${ }^{209}$, S. Yanagita ${ }^{176}$, L. Yang ${ }^{205}$, S. Yoo ${ }^{180}$, T. Yoshida ${ }^{176}$, T. Yoshikoshi ${ }^{2}$, P. Yu ${ }^{71}$, P. Yu ${ }^{85}$, A. Yusafzai ${ }^{59}$, M. Zacharias ${ }^{20}$, G. Zaharijas ${ }^{68}$, B. Zaldivar ${ }^{14}$, L. Zampieri ${ }^{76}$, R. Zanin ${ }^{219}$, R. Zanmar Sanchez $^{29}$,D. Zaric ${ }^{150}$, M. Zavrtanik ${ }^{68}$, D. Zavrtanik ${ }^{68}$, A.A. Zdziarski ${ }^{49}$, A. Zech ${ }^{20}$, H. Zechlin ${ }^{64}$, A. Zenin ${ }^{139}$, A. Zerwekh ${ }^{35}$, V.I. Zhdanov ${ }^{136}$, K. Ziętara ${ }^{169}$, A. Zink ${ }^{142}$, J. Ziółkowski ${ }^{49}$, V. Zitelli ${ }^{21}$, M. Živec ${ }^{68}$, A. Zmija ${ }^{142}$

1 : Centre for Space Research, North-West University, Potchefstroom, 2520, South Africa

2: Institute for Cosmic Ray Research, University of Tokyo, 5-1-5, Kashiwa-no-ha, Kashiwa, Chiba 277-8582, Japan

3: Pontificia Universidad Católica de Chile, Av. Libertador Bernardo O'Higgins 340, Santiago, Chile

4 : AIM, CEA, CNRS, Université Paris-Saclay, Université Paris Diderot, Sorbonne Paris Cité, CEA Paris-Saclay, IRFU/DAp, Bat 709, Orme des Merisiers, 91191 Gif-sur-Yvette, France

5 : Centre for Advanced Instrumentation, Dept. of Physics, Durham University, South Road, Durham DH1 3LE, United Kingdom

6: Port d’Informació Científica, Edifici D, Carrer de l'Albareda, 08193 Bellaterrra (Cerdanyola del Vallès), Spain

7: School of Physics and Astronomy, Monash University, Melbourne, Victoria 3800, Australia

8: Laboratoire Leprince-Ringuet, École Polytechnique (UMR 7638, CNRS/IN2P3, Institut Polytechnique de Paris), 91128 Palaiseau, France

9: Department of Physics, Columbia University, 538 West 120th Street, New York, NY 10027, USA

10 : University of Oslo, Department of Physics, Sem Saelandsvei 24 - PO Box 1048 Blindern, N-0316 Oslo, Norway

11 : EMFTEL department and IPARCOS, Universidad Complutense de Madrid, 28040 Madrid, Spain

12 : Instituto de Astrofísica de Andalucía-CSIC, Glorieta de la Astronomía s/n, 18008, Granada, Spain

13: Institute of Space Sciences (ICE-CSIC), and Institut d'Estudis Espacials de Catalunya (IEEC), and Institució Catalana de Recerca I Estudis Avançats (ICREA), Campus UAB, Carrer de Can Magrans, s/n 08193 Cerdanyola del Vallés, Spain

14 : Instituto de Física Teórica UAM/CSIC and Departamento de Física Teórica, Universidad Autónoma de Madrid, c/ Nicolás Cabrera 13-15, Campus de Cantoblanco UAM, 28049 Madrid, Spain

15 : Dublin Institute for Advanced Studies, 31 Fitzwilliam Place, Dublin 2, Ireland

16: Universidad Nacional Autónoma de México, Delegación Coyoacán, 04510 Ciudad de México, Mexico

17: University of Geneva - Département de physique nucléaire et corpusculaire, 24 rue du Général-Dufour, 1211 Genève 4, Switzerland 18: INFN Dipartimento di Scienze Fisiche e Chimiche - Università degli Studi dell'Aquila and Gran Sasso Science Institute, Via Vetoio 1, Viale Crispi 7, 67100 L'Aquila, Italy 
19: Instituto de Astronomia, Geofísico, e Ciências Atmosféricas - Universidade de São Paulo, Cidade Universitária, R. do Matão, 1226, CEP 05508-090, São Paulo, SP, Brazil

20 : LUTH, GEPI and LERMA, Observatoire de Paris, CNRS, PSL University, 5 place Jules Janssen, 92190, Meudon, France

21 : INAF - Osservatorio di Astrofisica e Scienza dello spazio di Bologna, Via Piero Gobetti 93/3, 40129 Bologna, Italy

22 : INAF - Osservatorio Astrofisico di Arcetri, Largo E. Fermi, 5 - 50125 Firenze, Italy

23 : INFN Sezione di Perugia and Università degli Studi di Perugia, Via A. Pascoli, 06123 Perugia, Italy

24 : INFN Sezione di Napoli, Via Cintia, ed. G, 80126 Napoli, Italy

25: INFN Sezione di Roma Tor Vergata, Via della Ricerca Scientifica 1, 00133 Rome, Italy

26 : Argonne National Laboratory, 9700 S. Cass Avenue, Argonne, IL 60439, USA

27 : Aix-Marseille Université, CNRS/IN2P3, CPPM, 163 Avenue de Luminy, 13288 Marseille cedex 09, France

28 : INAF - Osservatorio Astronomico di Roma, Via di Frascati 33, 00040, Monteporzio Catone, Italy

29: INAF - Osservatorio Astrofisico di Catania, Via S. Sofia, 78, 95123 Catania, Italy

30 : Grupo de Electronica, Universidad Complutense de Madrid, Av. Complutense s/n, 28040 Madrid, Spain

31: National Astronomical Research Institute of Thailand, 191 Huay Kaew Rd., Suthep, Muang, Chiang Mai, 50200, Thailand

32: Instituto de Astrofísica de Canarias and Departamento de Astrofísica, Universidad de La Laguna, La Laguna, Tenerife, Spain

33 : FZU - Institute of Physics of the Czech Academy of Sciences, Na Slovance 1999/2, 18221 Praha 8, Czech Republic

34 : Astronomical Institute of the Czech Academy of Sciences, Bocni II 1401 - 14100 Prague, Czech Republic

35 : CCTVal, Universidad Técnica Federico Santa María, Avenida España 1680, Valparaíso, Chile

36 : ETH Zurich, Institute for Particle Physics, Schafmattstr. 20, CH-8093 Zurich, Switzerland

37 : The University of Manitoba, Dept of Physics and Astronomy, Winnipeg, Manitoba R3T 2N2, Canada

38 : Department of Astronomy, University of Geneva, Chemin d'Ecogia 16, CH-1290 Versoix, Switzerland

39: Laboratoire Univers et Particules de Montpellier, Université de Montpellier, CNRS/IN2P3, CC 72, Place Eugène Bataillon, F-34095 Montpellier Cedex 5, France

40 : Centro Brasileiro de Pesquisas Físicas, Rua Xavier Sigaud 150, RJ 22290-180, Rio de Janeiro, Brazil

41 : Institut de Fisica d'Altes Energies (IFAE), The Barcelona Institute of Science and Technology, Campus UAB, 08193 Bellaterra

(Barcelona), Spain

42: University of Groningen, KVI - Center for Advanced Radiation Technology, Zernikelaan 25, 9747 AA Groningen, The Netherlands

43: School of Physics, University of New South Wales, Sydney NSW 2052, Australia

44 : INAF - Osservatorio Astrofisico di Torino, Strada Osservatorio 20, 10025 Pino Torinese (TO), Italy

45 : Univ. Savoie Mont Blanc, CNRS, Laboratoire d'Annecy de Physique des Particules - IN2P3, 74000 Annecy, France

46 : Department of Physics, TU Dortmund University, Otto-Hahn-Str. 4, 44221 Dortmund, Germany

47 : University of Zagreb, Faculty of electrical engineering and computing, Unska 3, 10000 Zagreb, Croatia

48 : University of Namibia, Department of Physics, 340 Mandume Ndemufayo Ave., Pioneerspark, Windhoek, Namibia

49: Nicolaus Copernicus Astronomical Center, Polish Academy of Sciences, ul. Bartycka 18, 00-716 Warsaw, Poland

50 : Universität Hamburg, Institut für Experimentalphysik, Luruper Chaussee 149, 22761 Hamburg, Germany

51: Graduate School of Science, University of Tokyo, 7-3-1 Hongo, Bunkyo-ku, Tokyo 113-0033, Japan

52 : Deutsches Elektronen-Synchrotron, Platanenallee 6, 15738 Zeuthen, Germany

53: Max-Planck-Institut für Kernphysik, Saupfercheckweg 1, 69117 Heidelberg, Germany

54 : RIKEN, Institute of Physical and Chemical Research, 2-1 Hirosawa, Wako, Saitama, 351-0198, Japan

55: INFN Sezione di Padova and Università degli Studi di Padova, Via Marzolo 8, 35131 Padova, Italy

56 : Escuela Politécnica Superior de Jaén, Universidad de Jaén, Campus Las Lagunillas s/n, Edif. A3, 23071 Jaén, Spain

57 : Department of Physics and Electrical Engineering, Linnaeus University, 35195 Växjö, Sweden

58: University of the Witwatersrand, 1 Jan Smuts Avenue, Braamfontein, 2000 Johannesburg, South Africa

59 : Institut für Theoretische Physik, Lehrstuhl IV: Plasma-Astroteilchenphysik, Ruhr-Universität Bochum, Universitätsstraße 150, 44801 Bochum, Germany

60 : Faculty of Physics and Applied Computer Science, University of Lódź, ul. Pomorska 149-153, 90-236 Lódź, Poland

61 : INAF - Istituto di Astrofisica Spaziale e Fisica Cosmica di Milano, Via A. Corti 12, 20133 Milano, Italy

62 : INFN and Università degli Studi di Siena, Dipartimento di Scienze Fisiche, della Terra e dell'Ambiente (DSFTA), Sezione di Fisica, Via Roma 56, 53100 Siena, Italy

63 : Center for Astrophysics | Harvard \& Smithsonian, 60 Garden St, Cambridge, MA 02180, USA

64 : INFN Sezione di Torino, Via P. Giuria 1, 10125 Torino, Italy

65 : Finnish Centre for Astronomy with ESO, University of Turku, Finland, FI-20014 University of Turku, Finland

66 : Pidstryhach Institute for Applied Problems in Mechanics and Mathematics NASU, 3B Naukova Street, Lviv, 79060, Ukraine

67 : Bhabha Atomic Research Centre, Trombay, Mumbai 400085, India

68 : Center for Astrophysics and Cosmology, University of Nova Gorica, Vipavska 11c, 5270 Ajdovščina, Slovenia

69 : Institut für Astronomie und Astrophysik, Universität Tübingen, Sand 1, 72076 Tübingen, Germany

70 : Research School of Astronomy and Astrophysics, Australian National University, Canberra ACT 0200, Australia

71 : Department of Physics and Astronomy, University of California, Los Angeles, CA 90095, USA

72: INFN Sezione di Bari and Politecnico di Bari, via Orabona 4, 70124 Bari, Italy

73 : Laboratoire de Physique des 2 infinis, Irene Joliot-Curie,IN2P3/CNRS, Université Paris-Saclay, Université de Paris, 15 rue Georges Clemenceau, 91406 Orsay, Cedex, France 
74 : INFN Sezione di Pisa, Largo Pontecorvo 3, 56217 Pisa, Italy

75 : IRFU/DEDIP, CEA, Université Paris-Saclay, Bat 141, 91191 Gif-sur-Yvette, France

76 : INAF - Osservatorio Astronomico di Padova, Vicolo dell'Osservatorio 5, 35122 Padova, Italy

77 : INAF - Osservatorio Astronomico di Palermo "G.S. Vaiana", Piazza del Parlamento 1, 90134 Palermo, Italy

78 : School of Physics, University of Sydney, Sydney NSW 2006, Australia

79 : Sorbonne Université, Université Paris Diderot, Sorbonne Paris Cité, CNRS/IN2P3, Laboratoire de Physique Nucléaire et de Hautes Energies, LPNHE, 4 Place Jussieu, F-75005 Paris, France

80 : Instituto de Física de São Carlos, Universidade de São Paulo, Av. Trabalhador São-carlense, 400 - CEP 13566-590, São Carlos, SP, Brazil

81 : Departament de Física Quàntica i Astrofísica, Institut de Ciències del Cosmos, Universitat de Barcelona, IEEC-UB, Martí i Franquès, 1, 08028, Barcelona, Spain

82 : Department of Physics, Washington University, St. Louis, MO 63130, USA

83 : Saha Institute of Nuclear Physics, Bidhannagar, Kolkata-700 064, India

84 : INAF - Osservatorio Astronomico di Capodimonte, Via Salita Moiariello 16, 80131 Napoli, Italy

85 : Université de Paris, CNRS, Astroparticule et Cosmologie, 10, rue Alice Domon et Léonie Duquet, 75013 Paris Cedex 13, France

86 : Astronomy Department of Faculty of Physics, Sofia University, 5 James Bourchier Str., 1164 Sofia, Bulgaria

87 : Institut de Recherche en Astrophysique et Planétologie, CNRS-INSU, Université Paul Sabatier, 9 avenue Colonel Roche, BP 44346, 31028 Toulouse Cedex 4, France

88 : School of Physics and Astronomy, University of Minnesota, 116 Church Street S.E. Minneapolis, Minnesota 55455-0112, USA

89: IRFU, CEA, Université Paris-Saclay, Bât 141, 91191 Gif-sur-Yvette, France

90 : INAF - Istituto di Radioastronomia, Via Gobetti 101, 40129 Bologna, Italy

91 : INAF - Istituto di Astrofisica Spaziale e Fisica Cosmica di Palermo, Via U. La Malfa 153, 90146 Palermo, Italy

92: Astronomical Observatory, Department of Physics, University of Warsaw, Aleje Ujazdowskie 4, 00478 Warsaw, Poland

93: Armagh Observatory and Planetarium, College Hill, Armagh BT61 9DG, United Kingdom

94 : INFN Sezione di Catania, Via S. Sofia 64, 95123 Catania, Italy

95 : INAF - Osservatorio Astronomico di Brera, Via Brera 28, 20121 Milano, Italy

96 : Kavli Institute for Particle Astrophysics and Cosmology, Department of Physics and SLAC National Accelerator Laboratory, Stanford University, 2575 Sand Hill Road, Menlo Park, CA 94025, USA

97 : Universidade Cruzeiro do Sul, Núcleo de Astrofísica Teórica (NAT/UCS), Rua Galvão Bueno 8687, Bloco B, sala 16, Libertade 01506-000 - São Paulo, Brazil

98 : Universidad de Valparaíso, Blanco 951, Valparaiso, Chile

99: INAF - Istituto di Astrofisica e Planetologia Spaziali (IAPS), Via del Fosso del Cavaliere 100, 00133 Roma, Italy

100 : Lund Observatory, Lund University, Box 43, SE-22100 Lund, Sweden

101 : The Henryk Niewodniczański Institute of Nuclear Physics, Polish Academy of Sciences, ul. Radzikowskiego 152, 31-342 Cracow, Poland

102 : Escola de Engenharia de Lorena, Universidade de São Paulo, Área I - Estrada Municipal do Campinho, s/nº, CEP 12602-810, Pte. Nova, Lorena, Brazil

103 : INFN Sezione di Trieste and Università degli Studi di Udine, Via delle Scienze 208, 33100 Udine, Italy

104 : Palacky University Olomouc, Faculty of Science, RCPTM, 17. listopadu 1192/12, 77146 Olomouc, Czech Republic

105 : Max-Planck-Institut für Physik, Föhringer Ring 6, 80805 München, Germany

106 : CENBG, Univ. Bordeaux, CNRS-IN2P3, UMR 5797, 19 Chemin du Solarium, CS 10120, F-33175 Gradignan Cedex, France

107 : Dublin City University, Glasnevin, Dublin 9, Ireland

108 : Dipartimento di Fisica - Universitá degli Studi di Torino, Via Pietro Giuria 1 - 10125 Torino, Italy

109: Tata Institute of Fundamental Research, Homi Bhabha Road, Colaba, Mumbai 400005, India

110 : Universitá degli Studi di Napoli "Federico II" - Dipartimento di Fisica "E. Pancini", Complesso universitario di Monte Sant’Angelo, Via Cintia - 80126 Napoli, Italy

111 : Oskar Klein Centre, Department of Physics, University of Stockholm, Albanova, SE-10691, Sweden

112 : Yale University, Department of Physics and Astronomy, 260 Whitney Avenue, New Haven, CT 06520-8101, USA

113 : CIEMAT, Avda. Complutense 40, 28040 Madrid, Spain

114 : University of Oxford, Department of Physics, Denys Wilkinson Building, Keble Road, Oxford OX1 3RH, United Kingdom

115: School of Physics \& Astronomy, University of Southampton, University Road, Southampton SO17 1BJ, United Kingdom

116 : Department of Physics and Technology, University of Bergen, Museplass 1, 5007 Bergen, Norway

117: Western Sydney University, Locked Bag 1797, Penrith, NSW 2751, Australia

118: School of Physical Sciences, University of Adelaide, Adelaide SA 5005, Australia

119: INFN Sezione di Roma La Sapienza, P.le Aldo Moro, 2 - 00185 Roma, Italy

120 : INFN Sezione di Bari, via Orabona 4, 70126 Bari, Italy

121 : University of Rijeka, Department of Physics, Radmile Matejcic 2, 51000 Rijeka, Croatia

122 : Institute for Theoretical Physics and Astrophysics, Universität Würzburg, Campus Hubland Nord, Emil-Fischer-Str. 31, 97074 Würzburg, Germany

123: Universidade Federal Do Paraná - Setor Palotina, Departamento de Engenharias e Exatas, Rua Pioneiro, 2153, Jardim Dallas, CEP: 85950-000 Palotina, Paraná, Brazil 
124 : Dept. of Physics and Astronomy, University of Leicester, Leicester, LE1 7RH, United Kingdom

125 : Univ. Grenoble Alpes, CNRS, IPAG, 414 rue de la Piscine, Domaine Universitaire, 38041 Grenoble Cedex 9, France

126 : National Centre for nuclear research (Narodowe Centrum Badań Jądrowych), Ul. Andrzeja Sołtana7, 05-400 Otwock, Świerk, Poland

127 : Enrico Fermi Institute, University of Chicago, 5640 South Ellis Avenue, Chicago, IL 60637, USA

128 : Institut für Physik \& Astronomie, Universität Potsdam, Karl-Liebknecht-Strasse 24/25, 14476 Potsdam, Germany

129 : Department of Physics and Astronomy, Iowa State University, Zaffarano Hall, Ames, IA 50011-3160, USA

130 : School of Physics, Aristotle University, Thessaloniki, 54124 Thessaloniki, Greece

131 : King's College London, Strand, London, WC2R 2LS, United Kingdom

132 : Escola de Artes, Ciências e Humanidades, Universidade de São Paulo, Rua Arlindo Bettio, CEP 03828-000, 1000 São Paulo, Brazil

133 : Dept. of Astronomy \& Astrophysics, Pennsylvania State University, University Park, PA 16802, USA

134 : National Technical University of Athens, Department of Physics, Zografos 9, 15780 Athens, Greece

135 : University of Wisconsin, Madison, 500 Lincoln Drive, Madison, WI, 53706, USA

136: Astronomical Observatory of Taras Shevchenko National University of Kyiv, 3 Observatorna Street, Kyiv, 04053, Ukraine

137 : Department of Physics, Purdue University, West Lafayette, IN 47907, USA

138 : Unitat de Física de les Radiacions, Departament de Física, and CERES-IEEC, Universitat Autònoma de Barcelona, Edifici C3, Campus UAB, 08193 Bellaterra, Spain

139 : Institute for Space-Earth Environmental Research, Nagoya University, Chikusa-ku, Nagoya 464-8601, Japan

140 : Department of Physical Science, Hiroshima University, Higashi-Hiroshima, Hiroshima 739-8526, Japan

141 : Department of Physics, Nagoya University, Chikusa-ku, Nagoya, 464-8602, Japan

142 : Friedrich-Alexander-Universität Erlangen-Nürnberg, Erlangen Centre for Astroparticle Physics (ECAP), Erwin-Rommel-Str. 1, 91058 Erlangen, Germany

143 : Santa Cruz Institute for Particle Physics and Department of Physics, University of California, Santa Cruz, 1156 High Street, Santa Cruz, CA 95064, USA

144 : IRFU / DIS, CEA, Université de Paris-Saclay, Bat 123, 91191 Gif-sur-Yvette, France

145 : INFN Sezione di Trieste and Università degli Studi di Trieste, Via Valerio 2 I, 34127 Trieste, Italy

146 : School of Physics \& Center for Relativistic Astrophysics, Georgia Institute of Technology, 837 State Street, Atlanta, Georgia, 30332-0430, USA

147 : Alikhanyan National Science Laboratory, Yerevan Physics Institute, 2 Alikhanyan Brothers St., 0036, Yerevan, Armenia

148 : INAF - Telescopio Nazionale Galileo, Roche de los Muchachos Astronomical Observatory, 38787 Garafia, TF, Italy

149: INFN Sezione di Bari and Università degli Studi di Bari, via Orabona 4, 70124 Bari, Italy

150 : University of Split - FESB, R. Boskovica 32, 21000 Split, Croatia

151 : Universidad Andres Bello, República 252, Santiago, Chile

152: Academic Computer Centre CYFRONET AGH, ul. Nawojki 11, 30-950 Cracow, Poland

153 : University of Liverpool, Oliver Lodge Laboratory, Liverpool L69 7ZE, United Kingdom

154 : Department of Physics, Yamagata University, Yamagata, Yamagata 990-8560, Japan

155 : Astronomy Department, Adler Planetarium and Astronomy Museum, Chicago, IL 60605, USA

156 : Faculty of Management Information, Yamanashi-Gakuin University, Kofu, Yamanashi 400-8575, Japan

157 : Department of Physics, Tokai University, 4-1-1, Kita-Kaname, Hiratsuka, Kanagawa 259-1292, Japan

158 : Centre for Astrophysics Research, Science \& Technology Research Institute, University of Hertfordshire, College Lane, Hertfordshire AL10 9AB, United Kingdom

159: Cherenkov Telescope Array Observatory, Saupfercheckweg 1, 69117 Heidelberg, Germany

160 : Tohoku University, Astronomical Institute, Aobaku, Sendai 980-8578, Japan

161 : Department of Physics, Rikkyo University, 3-34-1 Nishi-Ikebukuro, Toshima-ku, Tokyo, Japan

162 : Department of Physics and Astronomy and the Bartol Research Institute, University of Delaware, Newark, DE 19716, USA

163 : Institut für Astro- und Teilchenphysik, Leopold-Franzens-Universität, Technikerstr. 25/8, 6020 Innsbruck, Austria

164 : Department of Physics and Astronomy, University of Utah, Salt Lake City, UT 84112-0830, USA

165 : IMAPP, Radboud University Nijmegen, P.O. Box 9010, 6500 GL Nijmegen, The Netherlands

166 : Josip Juraj Strossmayer University of Osijek, Trg Ljudevita Gaja 6, 31000 Osijek, Croatia

167 : Department of Earth and Space Science, Graduate School of Science, Osaka University, Toyonaka 560-0043, Japan

168 : Yukawa Institute for Theoretical Physics, Kyoto University, Kyoto 606-8502, Japan

169 : Astronomical Observatory, Jagiellonian University, ul. Orla 171, 30-244 Cracow, Poland

170 : Landessternwarte, Zentrum für Astronomie der Universität Heidelberg, Königstuhl 12, 69117 Heidelberg, Germany

171 : University of Alabama, Tuscaloosa, Department of Physics and Astronomy, Gallalee Hall, Box 870324 Tuscaloosa, AL 354870324, USA

172 : Department of Physics, University of Bath, Claverton Down, Bath BA2 7AY, United Kingdom

173 : University of Iowa, Department of Physics and Astronomy, Van Allen Hall, Iowa City, IA 52242, USA

174 : Anton Pannekoek Institute/GRAPPA, University of Amsterdam, Science Park 9041098 XH Amsterdam, The Netherlands

175 : Faculty of Computer Science, Electronics and Telecommunications, AGH University of Science and Technology, Kraków, al. Mickiewicza 30, 30-059 Cracow, Poland 
176 : Faculty of Science, Ibaraki University, Mito, Ibaraki, 310-8512, Japan

177 : Faculty of Science and Engineering, Waseda University, Shinjuku, Tokyo 169-8555, Japan

178: Institute of Astronomy, Faculty of Physics, Astronomy and Informatics, Nicolaus Copernicus University in Toruń, ul. Grudziądzka 5, 87-100 Toruń, Poland

179: Graduate School of Science and Engineering, Saitama University, 255 Simo-Ohkubo, Sakura-ku, Saitama city, Saitama 338-8570, Japan

180 : Division of Physics and Astronomy, Graduate School of Science, Kyoto University, Sakyo-ku, Kyoto, 606-8502, Japan

181 : Centre for Quantum Technologies, National University Singapore, Block S15, 3 Science Drive 2, Singapore 117543, Singapore

182 : Institute of Particle and Nuclear Studies, KEK (High Energy Accelerator Research Organization), 1-1 Oho, Tsukuba, 305-0801, Japan

183 : Department of Physics and Astronomy, University of Sheffield, Hounsfield Road, Sheffield S3 7RH, United Kingdom

184 : Centro de Ciências Naturais e Humanas, Universidade Federal do ABC, Av. dos Estados, 5001, CEP: 09.210-580, Santo André SP, Brazil

185 : Dipartimento di Fisica e Astronomia, Sezione Astrofisica, Universitá di Catania, Via S. Sofia 78, I-95123 Catania, Italy

186 : Department of Physics, Humboldt University Berlin, Newtonstr. 15, 12489 Berlin, Germany

187 : Texas Tech University, 2500 Broadway, Lubbock, Texas 79409-1035, USA

188 : University of Zielona Góra, ul. Licealna 9, 65-417 Zielona Góra, Poland

189 : Institute for Nuclear Research and Nuclear Energy, Bulgarian Academy of Sciences, 72 boul. Tsarigradsko chaussee, 1784 Sofia, Bulgaria

190 : University of Białystok, Faculty of Physics, ul. K. Ciołkowskiego 1L, 15-254 Białystok, Poland

191 : Faculty of Physics, National and Kapodestrian University of Athens, Panepistimiopolis, 15771 Ilissia, Athens, Greece

192: Universidad de Chile, Av. Libertador Bernardo O’Higgins 1058, Santiago, Chile

193 : Hiroshima Astrophysical Science Center, Hiroshima University, Higashi-Hiroshima, Hiroshima 739-8526, Japan

194 : Department of Applied Physics, University of Miyazaki, 1-1 Gakuen Kibana-dai Nishi, Miyazaki, 889-2192, Japan

195 : School of Allied Health Sciences, Kitasato University, Sagamihara, Kanagawa 228-8555, Japan

196 : Departamento de Astronomía, Universidad de Concepción, Barrio Universitario S/N, Concepción, Chile

197 : Charles University, Institute of Particle \& Nuclear Physics, V Holešovičkách 2, 18000 Prague 8, Czech Republic

198 : Astronomical Observatory of Ivan Franko National University of Lviv, 8 Kyryla i Mephodia Street, Lviv, 79005, Ukraine

199 : Kobayashi-Maskawa Institute (KMI) for the Origin of Particles and the Universe, Nagoya University, Chikusa-ku, Nagoya 464-8602, Japan

200 : Graduate School of Technology, Industrial and Social Sciences, Tokushima University, Tokushima 770-8506, Japan

201 : Space Research Centre, Polish Academy of Sciences, ul. Bartycka 18A, 00-716 Warsaw, Poland

202 : Instituto de Física - Universidade de São Paulo, Rua do Matão Travessa R Nr.187 CEP 05508-090 Cidade Universitária, São Paulo, Brazil

203 : International Institute of Physics at the Federal University of Rio Grande do Norte, Campus Universitário, Lagoa Nova CEP 59078-970 Rio Grande do Norte, Brazil

204 : University College Dublin, Belfield, Dublin 4, Ireland

205 : Centre for Astro-Particle Physics (CAPP) and Department of Physics, University of Johannesburg, PO Box 524, Auckland Park 2006, South Africa

206 : Departamento de Física, Facultad de Ciencias Básicas, Universidad Metropolitana de Ciencias de la Educación, Santiago, Chile 207 : Núcleo de Formação de Professores - Universidade Federal de São Carlos, Rodovia Washington Luís, km 235 CEP 13565-905 SP-310 São Carlos - São Paulo, Brazil

208 : Physik-Institut, Universität Zürich, Winterthurerstrasse 190, 8057 Zürich, Switzerland

209 : Department of Physical Sciences, Aoyama Gakuin University, Fuchinobe, Sagamihara, Kanagawa, 252-5258, Japan

210: University of the Free State, Nelson Mandela Avenue, Bloemfontein, 9300, South Africa

211 : Faculty of Electronics and Information, Warsaw University of Technology, ul. Nowowiejska 15/19, 00-665 Warsaw, Poland

212 : Rudjer Boskovic Institute, Bijenicka 54, 10000 Zagreb, Croatia

213 : Department of Physics, Konan University, Kobe, Hyogo, 658-8501, Japan

214 : Kumamoto University, 2-39-1 Kurokami, Kumamoto, 860-8555, Japan

215 : University School for Advanced Studies IUSS Pavia, Palazzo del Broletto, Piazza della Vittoria 15, 27100 Pavia, Italy

216: Aalto University, Otakaari 1, 00076 Aalto, Finland

217: Agenzia Spaziale Italiana (ASI), 00133 Roma, Italy

218: Observatoire de la Cote d'Azur, Boulevard de l'Observatoire CS34229, 06304 Nice Cedex 4, Franc

219: CTAO gGmbH, Via Piero Gobetti 93/3, 40129 Bologna, Italy 